\title{
DISCOVERY OF BROAD SOFT X-RAY ABSORPTION LINES FROM THE QUASAR WIND IN PDS 456
}

\author{
J. N. Reeves ${ }^{1,2}$, V. Braito ${ }^{1,3}$, E. Nardini ${ }^{2}$, E. Behar ${ }^{4,5}$, P. T. O’Brien ${ }^{6}$, F. Tombesi ${ }^{5,7}$, T. J. TurneR ${ }^{8}$, And M. T. Costa ${ }^{2}$ \\ ${ }^{1}$ Center for Space Science and Technology, University of Maryland Baltimore County, 1000 Hilltop Circle, Baltimore, MD 21250, USA; jreeves@umbc.edu \\ ${ }^{2}$ Astrophysics Group, School of Physical and Geographical Sciences, Keele University, Keele, Staffordshire, ST5 5BG, UK; j.n.reeves@keele.ac.uk \\ ${ }^{3}$ INAF-Osservatorio Astronomico di Brera, Via Bianchi 46 I-23807 Merate (LC), Italy \\ ${ }^{4}$ Department of Physics, Technion, Haifa 32000, Israel \\ ${ }^{5}$ Department of Astronomy, University of Maryland, College Park, MD 20742, USA \\ ${ }^{6}$ Dept of Physics and Astronomy, University of Leicester, University Road, Leicester LE1 7RH, UK \\ ${ }^{7}$ Astrophysics Science Division, NASA/Goddard Space Flight Center, Greenbelt, MD 20771, USA \\ ${ }^{8}$ Department of Physics, University of Maryland Baltimore County, 1000 Hilltop Circle, Baltimore, MD 21250, USA \\ Received 2016 February 4; accepted 2016 April 12; published 2016 June 6
}

\begin{abstract}
High-resolution soft X-ray spectroscopy of the prototype accretion disk wind quasar, PDS 456, is presented. Here, the XMM-Newton reflection grating spectrometer spectra are analyzed from the large 2013-2014 XMM-Newton campaign, consisting of five observations of approximately $100 \mathrm{ks}$ in length. During the last observation (OBS. E), the quasar is at a minimum flux level, and broad absorption line (BAL) profiles are revealed in the soft X-ray band, with typical velocity widths of $\sigma_{\mathrm{v}} \sim 10,000 \mathrm{~km} \mathrm{~s}^{-1}$. During a period of higher flux in the third and fourth observations (OBS. C and D, respectively), a very broad absorption trough is also present above $1 \mathrm{keV}$. From fitting the absorption lines with models of photoionized absorption spectra, the inferred outflow velocities lie in the range $\sim 0.1-0.2 c$. The absorption lines likely originate from He and H-like neon and L-shell iron at these energies. A comparison with earlier archival data of PDS 456 also reveals a similar absorption structure near $1 \mathrm{keV}$ in a $40 \mathrm{ks}$ observation in 2001, and generally the absorption lines appear most apparent when the spectrum is more absorbed overall. The presence of the soft X-ray BALs is also independently confirmed by an analysis of the XMM-Newton EPIC spectra below $2 \mathrm{keV}$. We suggest that the soft X-ray absorption profiles could be associated with a lower ionization and possibly clumpy phase of the accretion disk wind, where the latter is known to be present in this quasar from its well-studied iron $\mathrm{K}$ absorption profile and where the wind velocity reaches a typical value of $0.3 c$.
\end{abstract}

Key words: black hole physics - quasars: individual (PDS 456) - X-rays: galaxies

\section{INTRODUCTION}

The masses of supermassive black holes (SMBHs, with $M_{\mathrm{BH}}=10^{6}-10^{9} M_{\odot}$ ) are known to correlate with galaxy bulge mass (Magorrian et al. 1998) and even more tightly with the stellar velocity dispersion on kiloparsec scales; this is the socalled $M-\sigma$ relation (Ferrarese \& Merritt 2000; Gebhardt et al. 2000). This implies a coevolution between SMBHs and their host galaxies, although the exact mechanism linking the two remained unclear. A potential mechanism responsible for this coevolution came with the discovery of extremely energetic outflows from the black holes powering the most luminous active galactic nuclei (AGNs) and quasars (Chartas et al. 2002, 2003; Pounds et al. 2003; Reeves et al. 2003; Tombesi et al. 2010; Gofford et al. 2013). At high redshifts, such winds would have provided the necessary mechanical feedback that both controlled the formation of stellar bulges and simultaneously regulated SMBH growth, leaving the observed $M-\sigma$ relation as a record of the process (Silk \& Rees 1998; King 2003; Di Matteo et al. 2005).

In the local universe $(z<0.3)$, the most powerful and bestcharacterized X-ray wind observed so far in an AGN is hosted by PDS 456 , a nearby $(z=0.184)$ radio-quiet quasar identified only in 1997 (Torres et al. 1997). PDS 456 is a remarkable object in many respects. The optical and near-infrared spectra show Balmer and Paschen lines with broad wings (full width at zero intensity of $>30,000 \mathrm{~km} \mathrm{~s}^{-1}$; Simpson et al. 1999), while in the Hubble Space Telescope (HST)/STIS UV spectrum the C Iv $\lambda 1549 \AA$ emission line is blueshifted by $\sim 5000 \mathrm{~km} \mathrm{~s}^{-1}$, and a tentative absorption trough extends from $\sim 14,000$ to $24,000 \mathrm{~km} \mathrm{~s}^{-1}$ blueward of the Ly $\alpha$ rest-frame energy (O'Brien et al. 2005). The bolometric luminosity of PDS 456, of the order of $L_{\mathrm{bol}}=10^{47} \mathrm{erg} \mathrm{s}^{-1}$ (Reeves et al. 2000; Yun et al. 2004), is more typical of a source at the peak of the quasar epoch $(z \sim 2-3)$, when AGN feedback is thought to have played a major role in the evolution of galaxies. No direct measurement is available for the mass of the central black hole, but this can be estimated from the SMBH-host galaxy scaling relations to be $\sim(1-2) \times 10^{9} M_{\odot}$ (Nardini et al. 2015), thus implying that the black hole in PDS 456 is accreting at a substantial fraction of the Eddington rate. Under these physical conditions, the photon momentum flux can contribute to the driving of massive accretion disk winds (several $M_{\odot} \mathrm{yr}^{-1}$ ), provided that the nuclear environment is sufficiently opaque to the continuum radiation (e.g., King 2010; see also Hagino et al. 2015).

The clear presence of strong absorption above $7 \mathrm{keV}$ in PDS 456 was revealed by a short (40 ks) XMM-Newton observation in 2001. If attributed to iron K-shell absorption, such a feature would have arisen in a high-velocity outflow, requiring a large column density of highly ionized matter (Reeves et al. 2003). An unusual absorption trough was also found in the soft X-ray band near $1 \mathrm{keV}$ (see also Behar et al. 2010). The detection of fast Fe $\mathrm{K}$ absorption has been confirmed in subsequent Suzaku campaigns in 2007, 2011, and 2013 , which implied an outflow velocity of $\sim 0.3 c$ (Reeves et al. 2009, 2014; Gofford et al. 2014). The broad, blueshifted emission and absorption profiles in the UV could then be potentially associated with the decelerating phase of the wind out to large scales. 
PDS 456 was observed again with XMM-Newton in a series of five observations between 2013 August and 2014 February, with the first four sequences carried out over about a month and the last one 6 months later, in order to sample the spectral variations over different timescales. All of the observations were complemented by the simultaneous high-energy spectra collected by $N U S T A R$, which provide a valuable broadband view that extends from the optical/UV to the hard X-ray. In this campaign, Nardini et al. (2015) were able to detect a persistent P-Cygni-like profile from highly ionized iron, thus establishing the wide-angle character of the disk wind in PDS 456. This proved to be important in accurately determining the overall energy budget of the outflow in PDS 456, made possible through establishing that the overall wind solid angle was a significant fraction of $4 \pi$ steradian. Thus the inner disk wind expels matter at rates close to Eddington, with a kinetic power a significant fraction of the bolometric output of the quasar, comfortably exceeding the typical values thought to be significant for quasar-mode feedback (Hopkins \& Elvis 2010). In many respects, PDS 456 strongly resembles the two fast outflows recently measured in two other luminous obscured quasars, IRAS F11119+3257 (Tombesi et al. 2015) and Mrk 231 (Feruglio et al. 2015). Here the initial accretion disk wind is likely critical in evacuating matter from the central regions of these systems during their postmerger phases, with the eventual fate of the gas seen at large (approximately kiloparsec) scales through massive $\left(\sim 1000 M_{\odot} \mathrm{yr}^{-1}\right)$, energyconserving molecular outflows.

However, to date there have been very few detections of fast AGN outflows in the soft X-ray band (see Gupta et al. 2013, 2015; Pounds 2014; Longinotti et al. 2015 for some possible recent examples). Here we present the soft X-ray spectroscopy of PDS 456 obtained from the XMM-Newton reflection grating spectrometer (RGS; den Herder et al. 2001) as part of the extended 2013-2014 campaign. In the subsequent analysis we will show the detection of broad soft X-ray absorption profiles in PDS 456, which may be potentially associated with the fast wind measured in the iron $\mathrm{K}$ band. In Section 2, we outline the $X M M-N e w t o n$ observations and data reduction. In Section 3 the overall form of the mean RGS spectrum is discussed, and Section 4 presents the detection of the soft X-ray absorption profiles in the individual RGS observations. In Section 5 photoionization modeling of the putative wind in the soft X-ray band is presented, and Section 6 discusses the origins of the absorbing gas from a clumpy, multiphase accretion disk wind. Throughout this work we assume the concordance cosmological values of $H_{0}=70 \mathrm{~km} \mathrm{~s}^{-1} \mathrm{Mpc}^{-1}$ and $\Omega_{\Lambda_{0}}=0.73$, and errors are quoted at $90 \%$ confidence $\left(\Delta \chi^{2}=2.7\right)$ for one parameter of interest. In the spectral analysis, we adopt a conversion between energy and wavelength of $E=(12.3984 \AA / \lambda) \mathrm{keV}$.

\section{XMM-Newton OBSERVATIONS OF PDS 456}

Five XMM-Newton observations of PDS 456, of at least $100 \mathrm{ks}$ in duration, were performed over a 6 month time period in 2013-2014. The EPIC-pn and MOS instruments were operating in Large Window mode and with the Thin filter applied. The observations are listed in Table 1 and are described in more detail in Nardini et al. (2015). The first four observations (hereafter OBS. A-D) were performed within a 4 week period (with separations of 4-10 days between observations), while the fifth observation (OBS. E) was performed about 5 months later and caught the AGN in the lowest flux state of the whole campaign (see Table 1). The XMM-Newton data have been processed and cleaned using the Science Analysis Software (sAS v14.0.0) and analyzed using standard software packages (FTOOLS v6.17, XSPEC v12.9).

For the scientific analysis of this paper, we concentrated on the RGS data, which have the highest spectral resolution in the soft X-ray band, and we compared the RGS results with the EPIC MOS and pn data for consistency. The RGS data have been reduced using the standard SAS task rgsproc and the most recent calibration files. High background time intervals have been filtered out, applying a threshold of 0.2 counts $\mathrm{s}^{-1}$ on the background event files. OBS. A was affected by severe telemetry issues, and about $28 \mathrm{ks}$ into the observation, even if the data were still recorded, the RGS telemetry became corrupted. The main effect is not a simple loss of the data but an incorrect count rate for each of the RGS spectra. Because it was not possible to recover the ODF files and because the subsequent RGS spectra were not suitable for analysis, hereafter we concentrate only on OBS. B-E from this campaign. Nonetheless, during OBS. A, from an analysis of the unaffected EPIC spectra, PDS 456 was caught in a bright and less obscured state, which was mainly featureless in the soft X-ray band (see Nardini et al. 2015).

For each of the remaining four observations, we first checked that the RGS 1 and RGS 2 spectra were in good agreement, typically to within the $3 \%$ level, and we subsequently combined them with the SAS task rgscombine. The spectra were analyzed over the $0.45-2.0 \mathrm{keV}$ energy range; below $0.45 \mathrm{keV}$ the spectra are noisy due to the Galactic absorption column toward PDS 456. During these observations, the two RGSs collected typically $\sim 8600-13,600$ net counts per observation (see Table 1 for details). Two of the observations (OBS. C and OBS. D), which were separated by only 4 days and show a very similar spectral shape and count rate, were further combined using rgscombine into a single spectrum (OBS. CD). Finally, in order to investigate the mean spectral properties with a higher signal-to-noise ratio $(\mathrm{S} / \mathrm{N})$ spectrum, we combined all four available observations obtained during this campaign.

In the initial spectral fitting, we first adopted a spectral binning of $\Delta \lambda=0.1 \AA$ for the RGS spectra, which just slightly undersamples the FWHM spectral resolution of $\Delta \lambda=0.06-0.08 \AA$. At this binning, the spectra have $>20$ counts per bin, and thus $\chi^{2}$ minimization was used for all of the spectral fitting. Note that in the subsequent analysis we also considered a finer binning (of $\Delta \lambda=0.05 \AA$ ) in order to search for any narrow features in the combined RGS $1+2$ spectra, as well as a coarser binning (of $\Delta \lambda=0.2 \AA$ ) for modeling the broad spectral features present in some of the individual sequences.

The XMM-Newton EPIC data were filtered for high background time intervals, which yields net exposure times as listed in Table 1. For the analysis we concentrated on the MOS spectra, as they offer a better spectral resolution in the soft band compared to the pn data; however, we did check the pn spectra for consistency in each observation. The MOS $1+2$ source and background spectra were extracted using a circular region with a radius of $35^{\prime \prime}$ and two circular regions with the same radius, respectively. Response matrices and ancillary response files at the source position were created using the sAs tasks arfgen and rmfgen. We then combined for each exposure the MOS 1 and 
Table 1

Summary of PDS 456 Observations

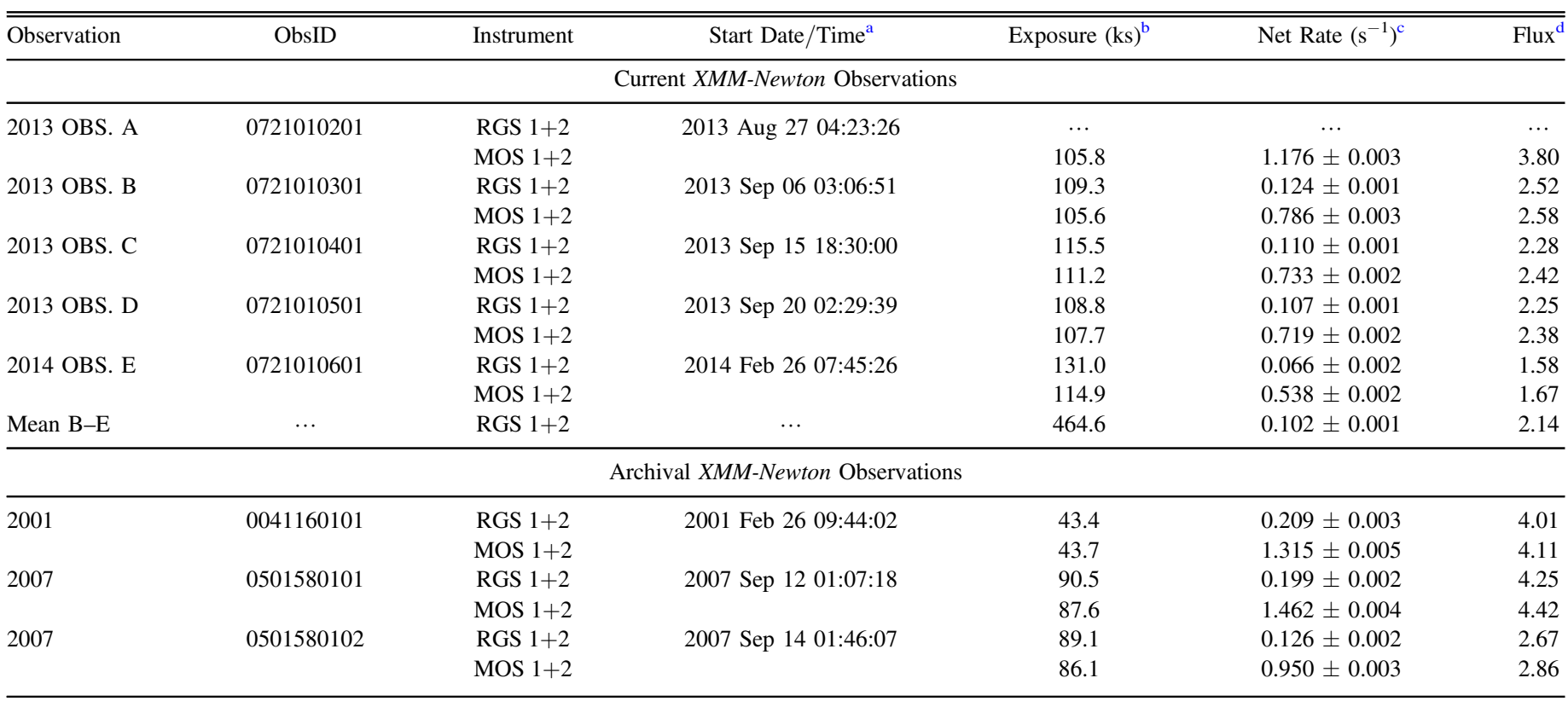

Notes.

a Observation start and end times are in UT.

b Net exposure time, after screening and deadtime correction, in ks.

${ }^{\mathrm{c}}$ Combined net count rate of RGS 1 and 2, or MOS 1 and 2 combined.

d Observed $0.5-2.0 \mathrm{keV}$ band fluxes, in units of $10^{-12} \mathrm{erg} \mathrm{cm}^{-2} \mathrm{~s}^{-1}$.

MOS 2 spectra, after verifying that the individual spectra were consistent. As for the RGS data, after checking that the MOS spectra for OBS. C and OBS. D were similar, we combined them into a single spectrum.

The MOS source spectra were then binned with constantenergy intervals of $15 \mathrm{eV}$. This oversamples the spectral resolution of the EPIC MOS detectors below $2 \mathrm{keV}$, where the FWHM is $\sim 50 \mathrm{eV}(60 \mathrm{eV})$ at $0.5 \mathrm{keV}(1 \mathrm{keV})$, by only a factor of 3-4. We note that adopting this binning allows us to simultaneously fit both the MOS and the RGS data with a similar sampling. The source is bright enough to collect between 0.54 and 0.79 counts s $^{-1}$ for the combined MOS $1+2$ spectra over the $0.5-2 \mathrm{keV}$ band.

In order to study the long-term spectral variability of the PDS 456 soft X-ray spectra, the RGS and EPIC spectra from the 2001 and 2007 archival observations were also extracted (see Table 1). The data were reduced in a way identical to the 2013-2014 observations. The 2001 observation consisted of a single exposure of $\sim 40 \mathrm{ks}$ duration, but at a higher overall count rate compared to the 2013-2014 observations, while the 2007 observations consisted of two sequences over two consecutive XMM-Newton orbits. These latter two 2007 spectra were consistent in spectral shape, with just a simple offset in flux between them, so they were combined to give a single RGS spectrum from 2007.

\section{INITIAL MEAN RGS SPECTRAL ANALYSIS}

Initially we combined all of the 2013-2014 RGS observations of PDS 456 (OBS. B through OBS. E) in order to construct a single, time-averaged spectrum. Although the spectrum of PDS 456 is time variable, the idea is to have an initial parameterization of both the continuum and local
Galactic absorption before proceeding with the analysis of the individual sequences. The mean RGS spectrum was fitted with a simple power-law plus blackbody continuum form, where the blackbody is required to account for the soft excess toward lower energies seen in this AGN. The mean spectrum was initially binned in constant-wavelength bins of $\Delta \lambda=0.1 \AA$ over the $0.45-2.0 \mathrm{keV}$ (or 6.2-28.0 $\AA$ ) range. $\mathrm{A}$ Galactic absorption column from $21 \mathrm{~cm}$ measurements of $N_{\mathrm{H}}=2.4 \times 10^{21} \mathrm{~cm}^{-2}$ (Dickey \& Lockman 1990; Kalberla et al. 2005) was adopted at first, modeled with the tbnew interstellar medium (ISM) absorption model in XSPEC using the cross sections and abundances of Wilms et al. (2000). However, in subsequent fits, the Galactic $N_{\mathrm{H}}$ value was allowed to vary, along with the relative abundance of oxygen (compared to $\mathrm{H}$ ) in order to ensure a good fit in the region around the neutral $\mathrm{O}$ edge.

This simple continuum form returned a photon index of $\Gamma=2.1 \pm 0.2$, with a blackbody temperature of $k T=102_{-10}^{+15} \mathrm{eV}$, while the Galactic column was found to be in excess of the $21 \mathrm{~cm}$ value with $N_{\mathrm{H}}=(3.9 \pm 0.6) \times 10^{21} \mathrm{~cm}^{-2}$. The resulting fit is shown in Figure 1, where the Galactic absorption model reproduces well the structure observed around the neutral $\mathrm{O}$ edge at $\sim 0.5 \mathrm{keV}$. The relative $\mathrm{O}$ abundance was found to be higher by a factor of $A_{\mathrm{O}}=1.27 \pm 0.12$ compared to the $\mathrm{O} / \mathrm{H}$ abundance of $4.9 \times 10^{-4}$ reported in Wilms et al. (2000). The absorbed $0.5-2.0 \mathrm{keV}$ soft $\mathrm{X}$-ray flux resulting from this model is $2.14 \times 10^{-12} \mathrm{erg} \mathrm{cm}^{-2} \mathrm{~s}^{-1}$. Note that if instead the solar abundances of Grevesse \& Sauval (1998) are adopted, which present a higher abundance of $\mathrm{O}$, then the relative $\mathrm{O}$ abundance is consistent with solar $\left(A_{\mathrm{O}}=0.95 \pm 0.09\right)$. While this model reproduces the overall shape of the soft 


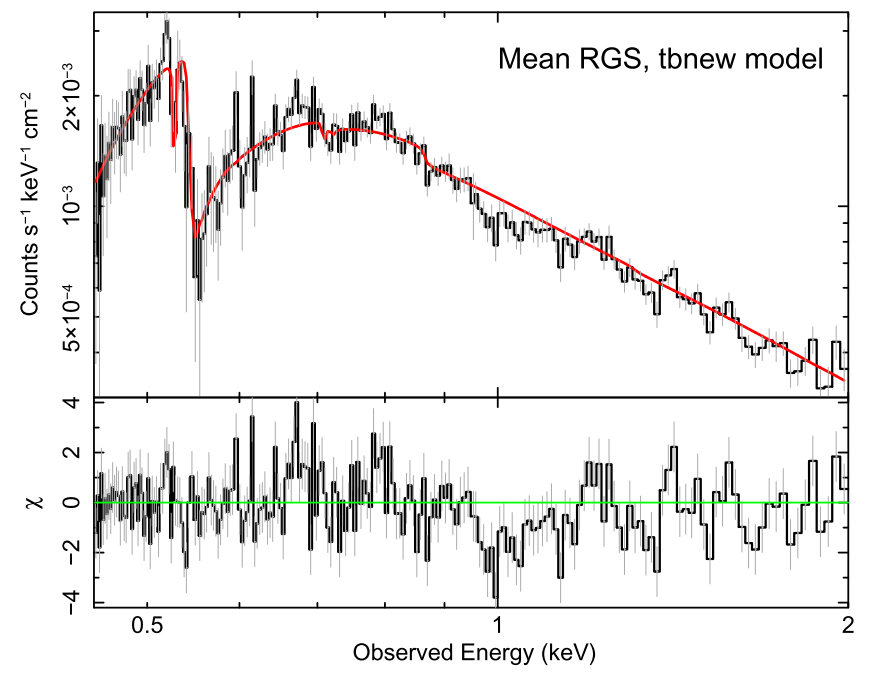

Figure 1. The summed RGS $1+2$ spectrum of PDS 456, taken from the mean of the B-E 2013-2014 observations. The $1 \sigma$ errors are shown in gray scale. The spectra have been rebinned with a constant resolution of $\Delta \lambda=0.1 \AA$ in wavelength and are plotted in the observed frame. This figure shows the RGS spectrum (upper panel) and residuals (in $\sigma$, lower panel) compared to the best-fit continuum model (solid red line) consisting of a power-law (of photon index $\Gamma \sim 2.1$ ) plus blackbody emission component. The Galactic absorption has been modeled with the tbnew ISM absorption model, as described in the text. Note the broad negative residuals present near $1 \mathrm{keV}$ in the observed frame.

$\mathrm{X}$-ray continuum, the fit statistic is relatively poor, with a resulting reduced chi-squared of $\chi^{2} / \nu=287.7 / 205$, suggesting the presence of additional spectral complexity. An almost identical fit is obtained if a somewhat different form for the soft excess is used, for example, a power-law plus Comptonizeddisk spectrum.

The mean spectrum was also binned at a finer resolution of $\Delta \lambda=0.05 \AA$ per bin in order to better sample the resolution of the RGS over the spectral range of interest. The motivation for this is to check whether there is any signature of a warm absorber from low-velocity, low to moderately ionized outflowing gas, which is commonly detected in the high-resolution soft X-ray spectra of nearby Seyfert 1 galaxies (Kaastra et al. 2000; Sako et al. 2001; Kaspi et al. 2002). Furthermore, any such low-velocity (and distant) component of a warm absorber would not be expected to strongly vary between the observations. Thus with the high $\mathrm{S} / \mathrm{N}$ of the mean $464 \mathrm{ks}$ net exposure, any narrow absorption (or emission) lines from distant and less variable photoionized gas should be readily apparent in the spectrum close to the expected rest-frame energies of the strongest atomic lines in the soft X-ray band. The residuals to the above absorbed power-law plus blackbody fit are shown in Figure 2, which is plotted in the rest frame of PDS 456. It is clear that, upon inspection of the spectra, no strong, narrow absorption or emission lines are observed close to the expected positions of the strongest lines, such as the $\mathrm{He} \alpha$ and $\mathrm{Ly} \alpha$ resonance lines of $\mathrm{O}, \mathrm{Ne}, \mathrm{Mg}$, or $\mathrm{Si}$, or at the energy of the Fe M-shell unresolved transition array (UTA).

In order to place a formal limit on the column of any warm absorber toward PDS 456, the spectrum was modeled with an XSTAR absorption grid with a turbulence velocity of $\sigma=300 \mathrm{~km} \mathrm{~s}^{-1}$, adopting solar abundances (Grevesse \& Sauval 1998) and allowing for a modest outflow velocity of up to $5000 \mathrm{~km} \mathrm{~s}^{-1}$. The above baseline continuum was used. The

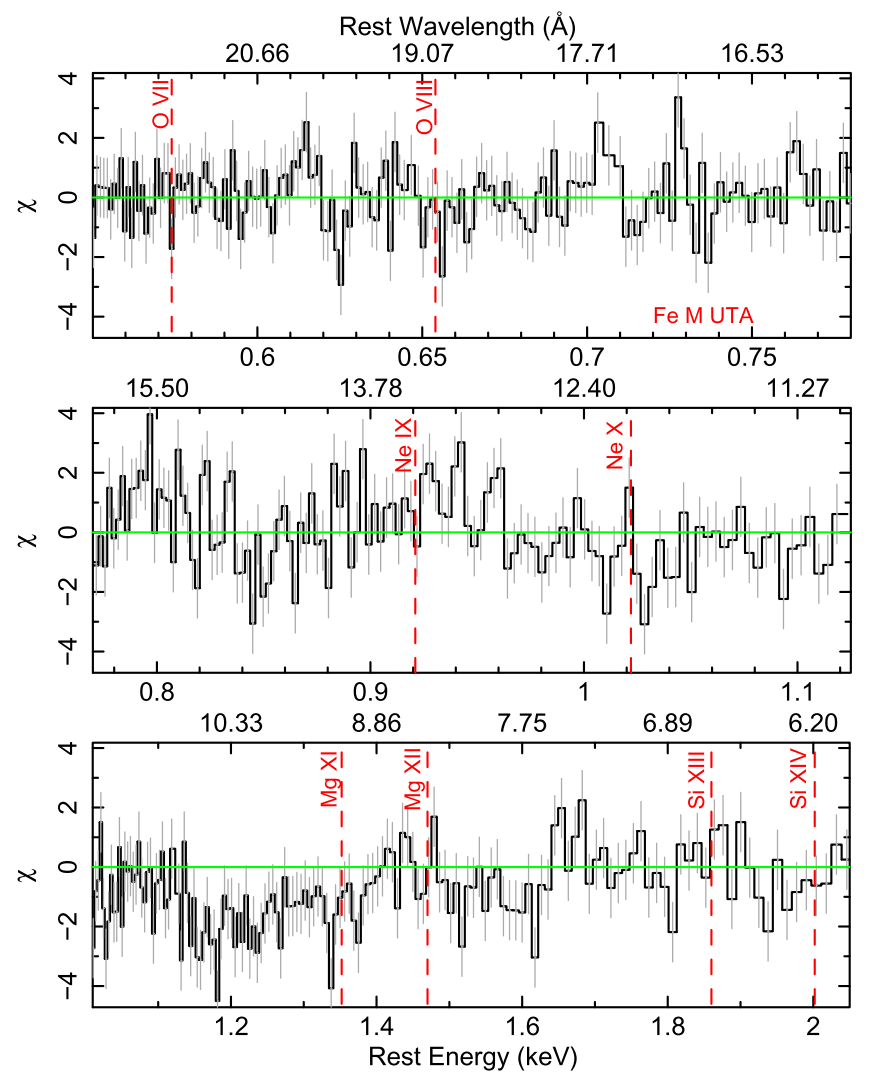

Figure 2. Deviations (in units $\sigma$ ) of the mean RGS data from the best-fit ISM absorption model obtained with the baseline TBNEW model, as plotted in Figure 1 and listed in Table 2. The $1 \sigma$ errors are shown in gray scale. The data have been rebinned with a finer resolution of $\Delta \lambda=0.05 \AA$. Rest energy (in $\mathrm{keV}$ ) is plotted along the lower $x$ axis, and rest wavelength (in $\AA$ ) along the upper axis. Dashed lines mark the expected positions of the resonance components of the strongest He-like $(\mathrm{He} \alpha)$ and $\mathrm{H}$-like $(\operatorname{Ly} \alpha)$ lines from abundant elements. The panels (from top to bottom) represent the residuals in the regions $\mathrm{O}, \mathrm{Ne}$, and $\mathrm{Mg} / \mathrm{Si}$, respectively. No significant narrow emission or absorption features are present near the expected positions of the strongest atomic lines, indicating the lack of a low-velocity warm absorber associated with PDS 456, but a clear broad residual structure is apparent, in particular in the $0.8-1.5 \mathrm{keV}$ range.

addition of this warm absorber component resulted in little improvement in the overall fit. Formally, we can place an upper limit on the column density of between $N_{\mathrm{H}}<0.6 \times 10^{20} \mathrm{~cm}^{-2}$ and $N_{\mathrm{H}}<1.7 \times 10^{20} \mathrm{~cm}^{-2}$ for an ionization parameter ${ }^{9}$ in the range $\log \xi=0-2$, which covers the typical range of ionization seen in the soft X-ray warm absorbers in Seyfert 1 's. Thus the presence of a low-velocity, low-ionization warm absorber appears to be ruled out in PDS 456.

Although no conventional signature of a warm absorber, in the form of narrow absorption lines, appears in these RGS spectra, the finely binned mean spectrum does reveal a broad residual structure over the energy range from 0.8 to $1.6 \mathrm{keV}$. Indeed, fitting with the above baseline continuum results in a very poor fit, with $\chi^{2} / \nu=573 / 406$ and a corresponding null-hypothesis probability of $8.6 \times 10^{-8}$. In the following sections we investigate whether these broad features may represent the soft X-ray signature of the known fast wind

\footnotetext{
9 The ionization parameter is defined as $\xi=L_{\mathrm{ion}} / n_{\mathrm{e}} r^{2}$, where $n_{\mathrm{e}}$ is the electron density, $r$ is the radial distance between the X-ray emitter and the ionized gas, and $L_{\text {ion }}$ is the ionizing luminosity over the 1-1000 Rydberg range. The units of $\xi$ are subsequently erg $\mathrm{cm} \mathrm{s}^{-1}$.
} 

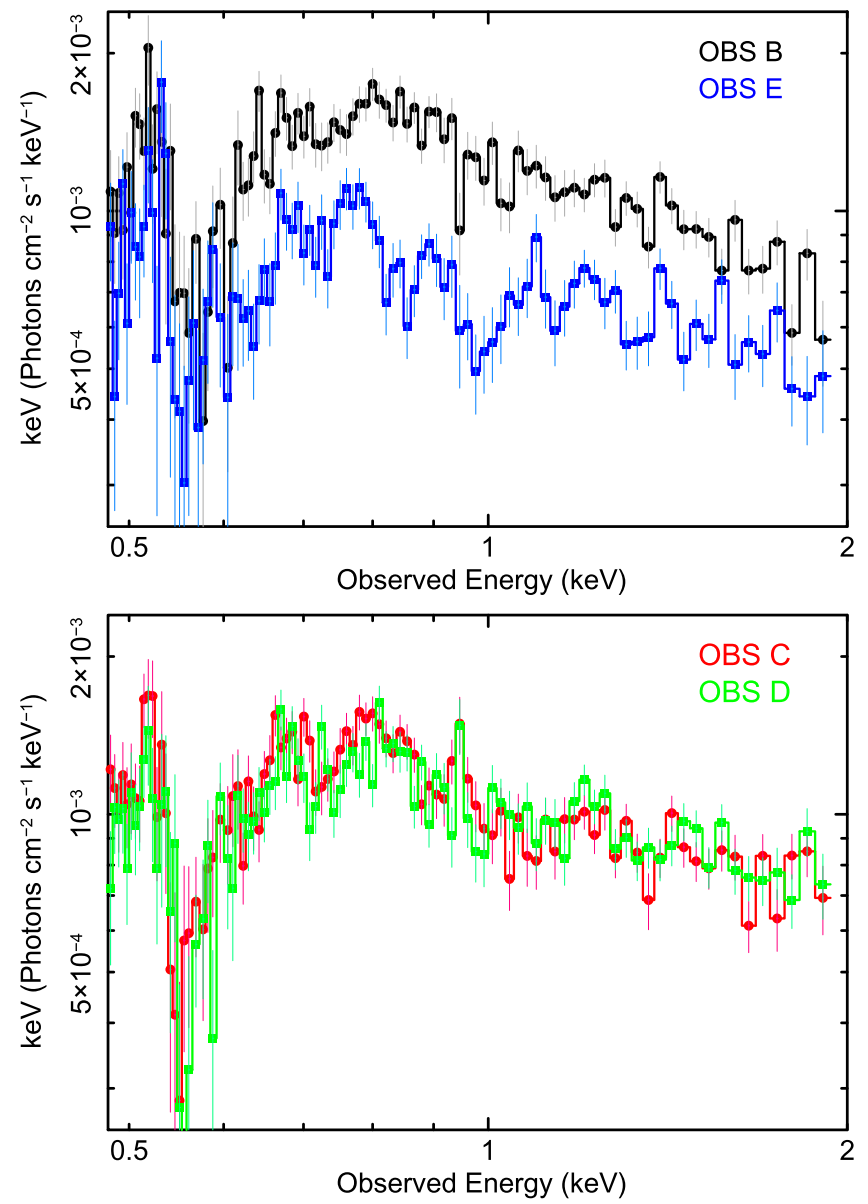

Figure 3. The four RGS spectral sequences of PDS 456 taken during the 2013-14 XMM-Newton campaign. The two panels show the fluxed spectra, unfolded against a simple power-law continuum. The upper plot shows the second (OBS. B, black circles) and highest flux sequence vs. the fifth (OBS. E, blue squares) and lowest flux sequences. A residual structure in the OBS. E spectrum is apparent around $1 \mathrm{keV}$ in the observed frame. The lower panel shows the third and fourth sequences (OBS. C and D, red circles and green squares, respectively). Taken only 4 days apart, these are consistent in form, so they have been combined for the spectral analysis. The strong absorption between 0.5 and $0.6 \mathrm{keV}$ in all the spectra is associated with the neutral $\mathrm{O}_{1}$ edge from our Galaxy.

toward PDS 456 detected at iron K (e.g., Reeves et al. 2009; Nardini et al. 2015). In the subsequent spectral fitting, we adopt the coarse spectral binning of $\Delta \lambda=0.2 \AA$ to model these broad profiles, as no narrow features are required by the data.

\section{ANALYSIS OF INDIVIDUAL RGS SEQUENCES}

We then proceeded to investigate each of the RGS spectra collected during the 2013-2014 campaign. A first inspection of the individual RGS spectra shows that there are remarkable differences between OBS. B (upper and black data points in the upper panel of Figure 3) and OBS. E (blue data points). The 0.5-2 keV spectral shapes appear broadly similar, with some variability of the intensity of the emerging continuum. In addition, the appearance of a deep trough at $\sim 1 \mathrm{keV}$ in OBS. E (observed frame) suggests that the variations could at least in part be caused by a variable soft X-ray absorber. On the other hand, the spectra obtained during OBS. C and OBS. D are almost identical (see Figure 3 lower panel) and are intermediate in flux between OBS. B and OBS. E. Thus in order to investigate the main driver of the soft $\mathrm{X}$-ray spectral variability, we proceeded to fit each of the main spectral states (OBS. B, OBS. CD, and OBS. E) independently. For the continuum we adopted the blackbody plus power-law continuum model found with the analysis of the mean RGS spectrum, allowing the $N_{\mathrm{H}}$ of the Galactic absorption to vary, as well as the continuum parameters.

We found that, while for OBS. CD and OBS. E the photon index of the primary power-law component is consistent with $\Gamma=2.1$, during OBS. B the spectrum requires a steeper power-law component $(\Gamma \sim 2.5 \pm 0.2)$. Statistically the fit is poor for most of the sequences when compared to the above continuum model: $\chi^{2} / \nu=149.9 / 100 \quad$ and $\chi^{2} / \nu=170.2 / 100$, for OBS. CD and OBS. E, respectively (see Table 2). In contrast, the continuum model can reproduce the overall shape of the RGS spectrum OBS. B $\left(\chi^{2} / \nu=131.2 / 100\right)$; indeed, as seen in Figure 4 (top panel), OBS. B appears almost featureless in the RGS.

However, the continuum model leaves clear residuals in the 0.9-1.3 keV energy range for OBS. CD and OBS. E. Broad and deep residuals are present in both of these spectra (Figure 4, second and third panels), where three main absorption troughs are detected. During OBS. CD a very broad absorption feature is present near $1.2 \mathrm{keV}$, while during OBS. E two absorption troughs emerge in the residuals at $\sim 1.0 \mathrm{keV}$ and $\sim 1.17 \mathrm{keV}$, respectively. In particular, a closer inspection of the residuals of the OBS. E spectrum to the best-fit continuum model (see Figure 5) unveils a complex absorption structure around $1 \mathrm{keV}$.

\subsection{Modeling and Identification of the Individual Profiles}

To parameterize these profiles, we added several Gaussian absorption and emission lines to the baseline continuum model. We imposed an initial selection criteria, whereby any line in the RGS spectra was added to the baseline model only if its addition yielded an improvement in the fit statistic of at least $\Delta \chi^{2}=9.2$ (for two interesting parameters) or $\Delta \chi^{2}=11.3$ (for three interesting parameters), equivalent to the $99 \%$ significance level in $\chi^{2}$ statistics. Furthermore, we impose an additional constraint, whereby any individual line in the RGS should be independently confirmed, corresponding to at least the same improvement in $\Delta \chi^{2}$ in the MOS (see Section 4.3). Thus, requiring a possible line detection in the RGS to be independently confirmed in the MOS, at a self-consistent energy and flux, reduces the likelihood that the line in question is spurious, due to photon noise. Subsequently the parameters of these Gaussian lines selected in the RGS are listed in Table 2, as well as the $F$-test probabilities associated with the addition of each line to the baseline model. Their statistical significances, which are typically confirmed at the $5 \sigma$ level or higher once the MOS data are accounted for, are evaluated more throughly in the Appendix.

As noted above, in OBS. CD we detected a strong $(\mathrm{EW}=42 \pm 13 \mathrm{eV})$ and very broad $(\sigma \sim 100 \mathrm{eV})$ absorption line at $E=1174 \pm 38 \mathrm{eV}$, with an apparent velocity width of $\sigma_{\mathrm{v}}=28000_{-9000}^{+13000} \mathrm{~km} \mathrm{~s}^{-1}$. We attempt to place a tentative identification on this absorption feature. The $\sim 1.2 \mathrm{keV}$ absorption line could be identified with blueshifted $\left(v_{\text {out }} \sim 0.2 c\right)$ Nex Ly $\alpha\left(E_{\text {Lab }}=1.022 \mathrm{keV}\right)$, perhaps blended with iron $\mathrm{L}$ absorption in the ionization range $\mathrm{Fe} x \mathrm{X}$-XxIV. Alternatively, associating this broad absorption line (BAL) with a single feature, but with a smaller blueshift, would require an identification with the Fe XxIV $2 s \rightarrow 3 p$ at $E_{\mathrm{Lab}}=1.167 \mathrm{keV}$. However, this would imply a single very 
Table 2

Best-Fit Parameters of the Strongest Gaussian Lines Fitted to RGS

\begin{tabular}{|c|c|c|c|c|c|c|c|}
\hline & $\begin{array}{l}\text { Rest Energy }{ }^{\mathrm{a}} \\
(\mathrm{eV})\end{array}$ & $\begin{array}{c}\sigma \\
(\mathrm{eV})\end{array}$ & $\begin{array}{c}\sigma_{\mathrm{v}} \\
\left(\mathrm{km} \mathrm{s}^{-1}\right)\end{array}$ & $\begin{array}{c}\text { Intensity } \\
\left(10^{-5} \mathrm{ph} \mathrm{cm}^{-2} \mathrm{~s}^{-1}\right)\end{array}$ & $\begin{array}{l}\text { EW } \\
(\mathrm{eV})\end{array}$ & $\Delta \chi^{2} / \Delta \nu$ & $P_{\mathrm{F}}^{\mathrm{b}}$ \\
\hline \multicolumn{8}{|c|}{ OBS. CD: No Gaussian lines, $\chi^{2} / \nu=170.2 / 100, N_{\mathrm{P}}=1.53 \times 10^{-5 \mathrm{c}}$} \\
\hline ABS1 & $846_{-6}^{+6}$ & $6.5_{-2.8}^{+6.0}$ & $2,300_{-1,000}^{+2,100}$ & $-5.0_{-2.4}^{+2.4}$ & $-4.6_{-2.2}^{+2.2}$ & $17.0 / 3$ & $8.2 \times 10^{-3}$ \\
\hline $\mathrm{ABS} 2$ & $1174_{-58}^{+47}$ & $109_{-35}^{+49}$ & $28,000_{-9,000}^{+13,000}$ & $-13.0_{-4.0}^{+4.0}$ & $-41_{-13}^{+13}$ & $25.2 / 3$ & $7.1 \times 10^{-4}$ \\
\hline \multicolumn{8}{|c|}{ OBS. E: No Gaussian lines, $\chi^{2} / \nu=149.9 / 100, N_{\mathrm{P}}=9.2 \times 10^{-4 c}$} \\
\hline ABS1 & $1016_{-18}^{+19}$ & $41_{-12}^{+14}$ & $12,000_{-3,500}^{+4,000}$ & $-11.2_{-5.4}^{+8.0}$ & $-27.3_{-13.2}^{+19.3}$ & $17.3 / 3$ & $1.4 \times 10^{-3}$ \\
\hline ABS2 & $1166_{-20}^{+19}$ & $41^{\mathrm{d}}$ & & $-6.7_{-2.8}^{+4.1}$ & $-31.3_{-13.0}^{+19.1}$ & $27.0 / 2$ & $9.0 \times 10^{-6}$ \\
\hline EMIS1 & $913_{-15}^{+16}$ & $18_{-8}^{+13}$ & $6,000_{-3,000}^{+4,000}$ & $5.2_{-3.2}^{+4.3}$ & $9.8_{-6.0}^{+8.1}$ & $11.6 / 3$ & 0.013 \\
\hline \multicolumn{8}{|c|}{ 2001: No Gaussian lines, $\chi^{2} / \nu=181.5 / 99, N_{\mathrm{P}}=8.4 \times 10^{-7 \mathrm{c}}$} \\
\hline ABS1 & $1061_{-11}^{+11}$ & $42_{-11}^{+13}$ & $11,900_{-3,100}^{+3,700}$ & $-40.6_{-16.8}^{+12.4}$ & $-37_{-15}^{+11}$ & $42.0 / 3$ & $1.32 \times 10^{-5}$ \\
\hline
\end{tabular}

Notes.

${ }^{a}$ Measured line energy in the PDS 456 rest frame.

b Null-hypothesis probability of any individual line, via $F$-test.

${ }^{c}$ Fit statistic and null-hypothesis probability against baseline continuum model (without Gaussian lines).

${ }^{\mathrm{d}}$ Denotes a tied parameter.

broad line seen in isolation, without a strong contribution from other Fe L lines, for example, Fe XxIII at $E_{\mathrm{Lab}}=1.125 \mathrm{keV}$ and lower ionization ions. Furthermore, as we will discuss in Section 5, modeling of this absorption profile with a grid of photoionized absorption spectra prefers the solution with a fast and highly ionized wind, noting that part of the large velocity width of the broad trough could be explained with a blend of these lines. Finally, a narrower absorption line is also apparent at $846 \mathrm{eV}$ in OBS. CD, and although it is much weaker than the broad $1.2 \mathrm{keV}$ profile, it is also independently confirmed in the MOS (see Section 4.3). While the identification of this isolated line is uncertain, we note that if it is tentatively associated with absorption from $\mathrm{O}$ VIII Ly $\alpha$ (at $E_{\mathrm{Lab}}=0.654 \mathrm{keV}$ ), its outflow velocity would be consistent with that found at iron $\mathrm{K}$, at $\sim 0.3 \mathrm{c}$.

The appearance of a complex soft X-ray absorption structure near $1 \mathrm{keV}$ in OBS. E is perhaps not unexpected, especially as this RGS sequence appears the most absorbed and at the lowest flux of all the 2013-2014 XMM-Newton data sets. As shown in Figure 5, two deep absorption lines (at $E \sim 1.01 \mathrm{keV}$ and $E \sim 1.17 \mathrm{keV}$ ) and an emission line at $E \sim 0.9 \mathrm{keV}$ are formally required by the data (see Table 2). Indeed, the addition of the two Gaussian absorption lines results in the fit statistic improving by $\Delta \chi^{2}=44.3$ for $\Delta \nu=5$. Assuming that the absorption line profiles have the same width, then the two lines are found to be resolved with a common width of $\sigma=41_{-12}^{+14} \mathrm{eV}\left(\right.$ or $\left.\sigma_{\mathrm{v}}=12000_{-3500}^{+4000} \mathrm{~km} \mathrm{~s}^{-1}\right)$.

One likely identification of the emission/absorption line pair in OBS. E at $913 \mathrm{eV}$ and $1016 \mathrm{eV}$ may be with $\mathrm{Ne}$ IX (at $E_{\mathrm{Lab}}=0.905 \mathrm{keV}$ ), which would then require the absorption line to be blueshifted with respect to the emission-line component. Alternatively, if the absorption at $1016 \mathrm{eV}$ is separately associated with $\mathrm{Ne} \times \mathrm{Ly} \alpha\left(\right.$ at $E_{\mathrm{Lab}}=1.022 \mathrm{keV}$ ), then this would require little or no velocity shift. However, the second absorption line at $1166 \mathrm{eV}$ only requires a blueshift if it is associated with $\mathrm{Ne} \times \mathrm{Ly} \alpha$; alternatively it could be associated with Fe XXIV $2 s \rightarrow 3 p$ without requiring a blueshift. Thus the precise identification of these $1 \mathrm{keV}$ absorption lines is difficult to determine on an ad hoc basis by fitting simple Gaussian profiles. Their most likely origin will be discussed further when we present the self-consistent photoionization modeling of the OBS. E spectrum in Section 5.3. Nonetheless, regardless of their possible identification, the detection of the broad absorption profiles in the soft X-ray band in both OBS. E and OBS. CD may suggest the presence of a new absorption zone with somewhat lower velocity or ionization compared to the well-known ionized wind established at iron $\mathrm{K}$.

\subsection{Comparison to the 2001 and 2007 XMM-Newton Observations}

Following the results of the 2013-2014 observations, we reanalyzed the RGS spectra collected during the past XMMNewton observations of PDS 456, when the quasar was observed in two extreme states: a highly obscured one (2001; Reeves et al. 2003) and an unobscured state (2007; Behar et al. 2010). For the continuum model we again adopted the best fit found with the analysis of the mean RGS spectrum, allowing the $N_{\mathrm{H}}$ of the Galactic absorption to vary as well as allowing the continuum parameters to adjust. For the 2001 observation we included an additional, neutral, partial covering absorber in the continuum model; indeed, during this observation, even though the intrinsic flux of PDS 456 is relatively high (see Table 1), the AGN appeared heavily obscured, with the presence of a strong spectral curvature over the $1-10 \mathrm{keV}$ band (Reeves et al. 2003). This curvature is especially evident in the EPIC MOS data (see Section 4.3). Without this absorber, the derived photon index, although poorly constrained, is extremely hard $(\Gamma \sim 1.5)$, and its extrapolation above $2 \mathrm{keV}$ lies well above the EPIC spectra. On the other hand, the 2007 spectrum required a steeper photon index $(\Gamma=2.4-2.5)$, indicating a lack of intrinsic absorption.

The residuals for the 2001 and 2007 RGS data to the baseline continuum model are shown in the two lower panels of Figure 4. Similarly to the OBS. E spectrum during the 2013-2014 campaign, the 2001 observation tracks the presence of the highly ionized wind. The presence of absorption near $1 \mathrm{keV}$ in the 2001 observation was first noted in this data set by 


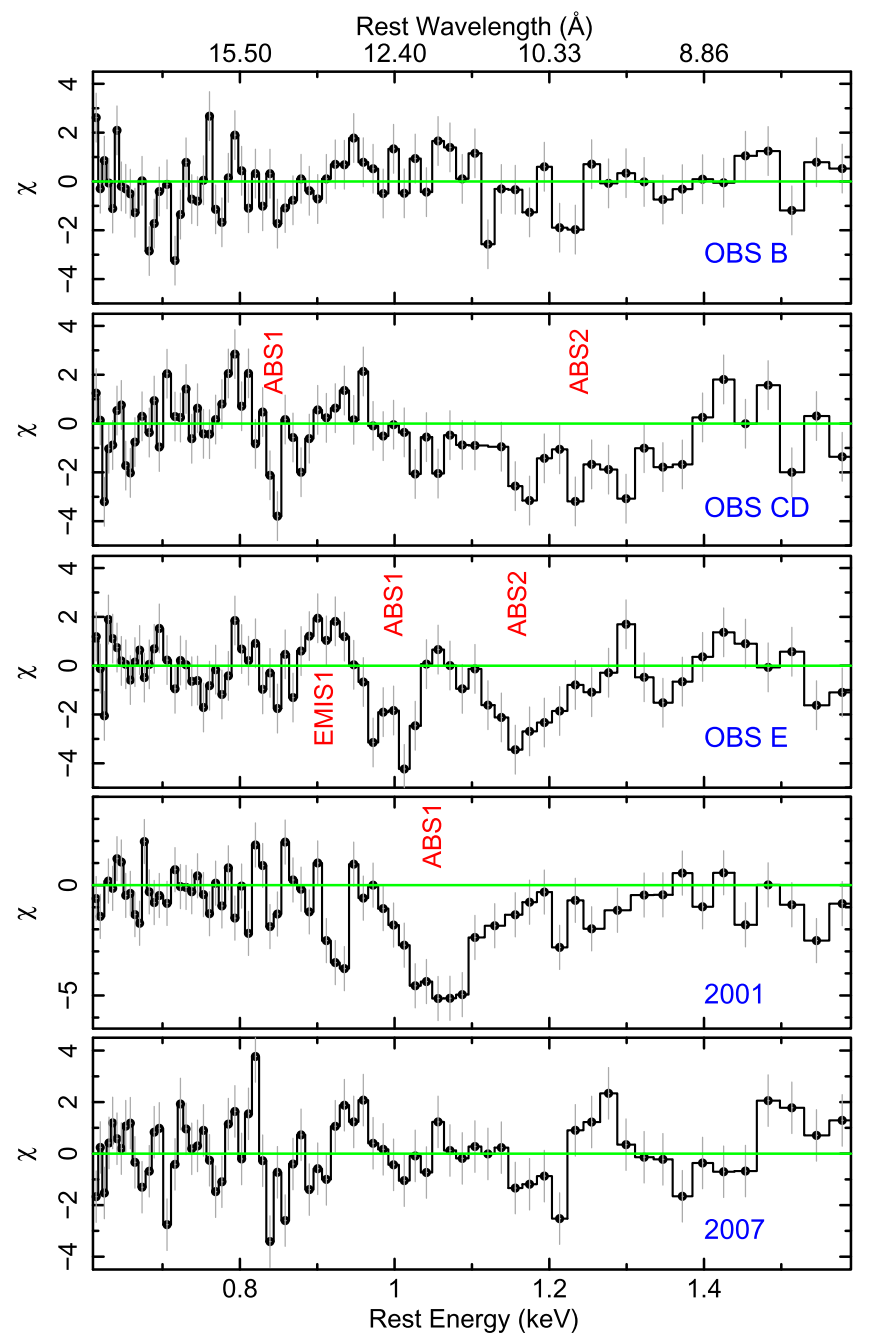

Figure 4. Panels showing the RGS spectral residuals to the baseline continuum model, for the 2013-2014 observations B, CD (combined), and E, as well as the earlier 2001 and 2007 RGS observations of PDS 456. The RGS spectra have been binned to $\Delta \lambda=0.2 \AA$, at approximately twice the spectral resolution, and are plotted in the rest frame of PDS 456 at $z=0.184$. Data points are shown as black circles, with $1 \sigma$ error bars shown in gray. Broad residuals are particularly apparent in some of the spectral sequences, such as in the form of absorption troughs seen at 1 and $1.17 \mathrm{keV}$ in OBS. E, a deep absorption dip centered at $1.06 \mathrm{keV}$ in the 2001 sequence, and a very broad absorption trough in OBS. CD near $1.2 \mathrm{keV}$. The significant features labeled above are those fitted with Gaussian profiles in Table 2.

Reeves et al. (2003). A highly significant, broad absorption profile is apparent in the residuals at $E=1061 \pm 11 \mathrm{eV}$, with an equivalent width of $\mathrm{EW} \sim 37 \mathrm{eV}$, while the improvement in the fit statistic upon adding this absorption line is $\Delta \chi^{2} / \Delta \nu=42.0 / 3$ (see Table 2). The profile is resolved with a width of $\sigma=42_{-11}^{+13} \mathrm{eV}$ ( or $\sigma_{\mathrm{v}}=11900_{-3100}^{+3700} \mathrm{~km} \mathrm{~s}^{-1}$ ), as per the similar broad profiles in OBS. E. As discussed above, one plausible identification is with mildly blueshifted $\mathrm{Ne} x \operatorname{Ly} \alpha$ (again notwithstanding any possible contribution from iron $\mathrm{L}$ absorption). At face value, this suggests the presence of a lower-velocity zone of the wind, which is investigated further in Section 5. In contrast to the 2001 spectrum, the 2007 observations show little evidence for intrinsic absorption and appear to be featureless (see also Behar et al. 2010).

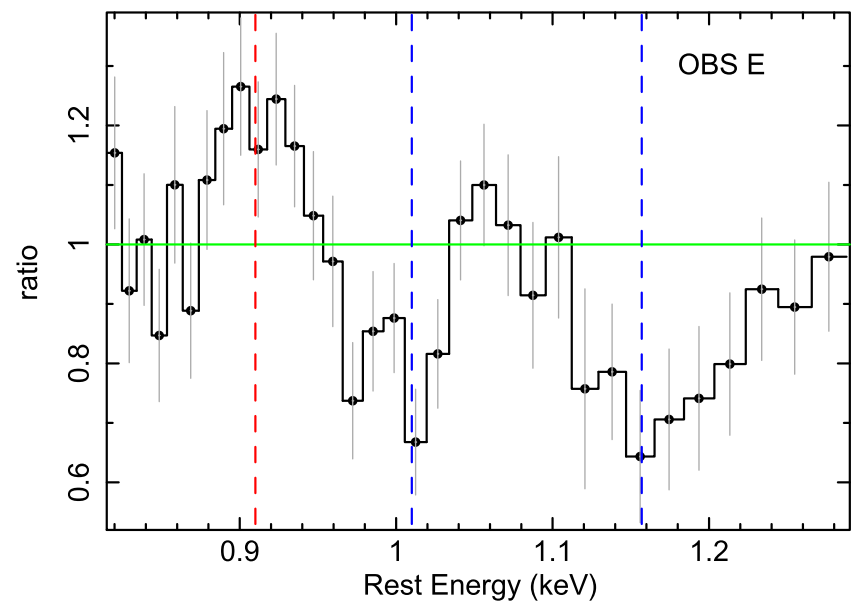

Figure 5. A zoom-in of the RGS spectra for OBS. E, showing the complex soft $\mathrm{X}$-ray features present near $1 \mathrm{keV}$. The spectrum is plotted as data/model residuals against the best-fit baseline continuum vs. rest-frame energy. The approximate energy centroids are marked as a red dashed line for the significant emission line and in blue for the significant absorption lines (see Table 2).

\subsection{Consistency Check with EPIC MOS Soft X-Ray Spectra}

In this section we perform a consistency check on the lines detected in the RGS with the EPIC MOS spectra. Indeed, given the strength and breadth of the absorption features detected in the RGS spectra, we may expect them to be detectable at the MOS CCD spectral resolution. We thus considered the combined MOS $1+2$ spectra for each observation and, while we fitted only the $0.5-2 \mathrm{keV}$ energy range where the soft X-ray features occur, we also checked that the best-fit models provide a good representation of the overall X-ray continuum to higher energies. The continuum model found from the analysis of the RGS spectra was adopted, but again allowing the parameters to adjust. As noted above, the 2001 MOS observation also shows a pronounced curvature between 1.2 and $2.0 \mathrm{keV}$, which is apparent in the residuals of this spectrum in Figure 6 and was accounted for by including a neutral, partial covering absorber.

The residuals of the five MOS spectra (OBS. B, CD, E, 2001, and 2007) to the best-fit continuum models are shown in Figure 6. Several absorption profiles are clearly visible (which are labeled on each of the corresponding spectral panels), and they are generally in good agreement with the features present in the RGS in Figure 4. In particular, the broad absorption profiles present during OBS. CD and OBS. E are confirmed at high significance, whereby both the broad profile at $\sim 1.2 \mathrm{keV}$ (OBS. CD) and the complex absorption structure in $0.9-1.2 \mathrm{keV}$ (OBS. E) emerge at high $\mathrm{S} / \mathrm{N}$ in the MOS data. Likewise, the broad absorption trough detected at $1.06 \mathrm{keV}$ in the 2001 data set is also confirmed in the MOS spectra, with self-consistent parameters. On the other hand, the featureless nature of the 2007 spectrum is also confirmed in the MOS, with no obvious residuals present in the spectrum. The results of the Gaussian line fitting to the MOS spectra are subsequently summarized in Table 3.

As a further check of the consistency between the RGS and MOS detections of the absorption lines, we generated and overlaid confidence contours for each of the lines that are independently detected in each of the RGS and MOS spectra. As an illustration, we show two of the examples from the OBS. $\mathrm{E}$ and 2001 sequences in Figure 7. The upper panel shows the $68 \%, 90 \%$, and $99 \%$ confidence contours (for two parameters 


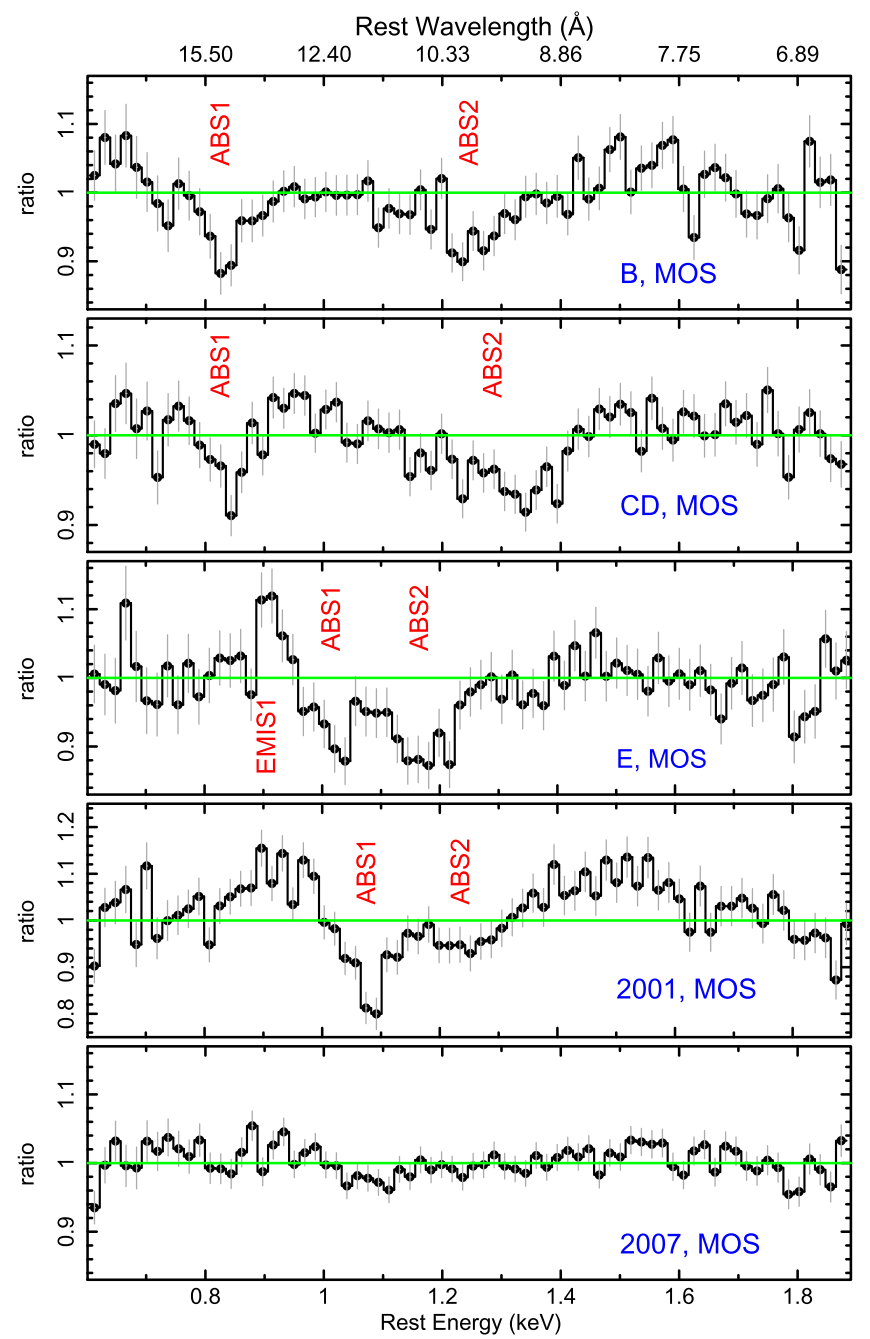

Figure 6. Data/model residuals in the soft X-ray band to the EPIC MOS observations, showing from top to bottom the 2013-2014 observations B, CD, and $\mathrm{E}$, as well as the earlier spectra from 2001 to 2007. The baseline continuum is a power-law plus blackbody component, absorbed by a Galactic column, as per the RGS. Several line features that are present in the RGS are also confirmed in the MOS spectra; in particular, note the two absorption troughs between 1.0 and $1.2 \mathrm{keV}$ in OBS. E, the deep absorption profile above $1 \mathrm{keV}$ in the 2001 observation, and the broad absorption trough above $1.2 \mathrm{keV}$ in OBS. CD. The significant features labeled above are those fitted with Gaussian profiles in Table 3 .

of interest) of line energy against flux for the $\sim 1.16 \mathrm{keV}$ absorption line detected in the OBS. E spectra. The contours show the close agreement between the two detectors, with the absorption line confirmed in both the RGS and MOS spectra. Generally the line fluxes are more tightly constrained in the MOS, which is likely due to the higher $\mathrm{S} / \mathrm{N}$ and better continuum determination afforded by the MOS spectra. The lower panel of Figure 7 shows the comparison between line energy and width (in terms of $\sigma$ ) for the $\sim 1 \mathrm{keV}$ broad absorption trough in the 2001 observation. The contours show that the broadening of the line is independently measured by both RGS and MOS, with a width of $\sigma \sim 40 \mathrm{eV}$ (or $\sigma=12,000 \mathrm{~km} \mathrm{~s}^{-1}$ ). The only marginal disagreement at the $90 \%$ level is in the line energy, which is offset by $\sim 20 \mathrm{eV}$, although this is likely to lie within the uncertainty of the absolute energy scale of the MOS detectors.

\section{MODELING WITH PHOTOIONIZED ABSORPTION MODELS}

Having established the consistent detection of absorption lines between the RGS and MOS spectra, in this next section we model the absorption (and any emission) from these spectra with a self-consistent XSTAR photoionization model (Kallman \& Bautista 2001). The goals of the modeling are to determine the likely identifications of the absorption profiles and to reveal the properties and possible outflow velocities of the soft X-ray absorption zones.

\subsection{Generation of Tabulated Photoionized Models}

In order to generate the XSTAR grids for the modeling, we used the input spectral energy distribution (SED) defined from a simultaneous fit to the XMM-Newton OM, EPIC, and NuSTAR data, taken from the 2013-2014 campaign. The details of the SED modeling can be found in Nardini et al. (2015); in summary, the SED can be parameterized by a double broken power law from between the optical-UV, UV to soft Xray, and X-ray portions of the spectrum. In the optical-UV band covered by the OM $(2-10 \mathrm{eV})$, the spectrum has $\Gamma \simeq 0.7$ (so rising in $\nu F_{\nu}$ space). Between the UV and soft $\mathrm{X}$-rays $(10-500 \mathrm{eV})$, the SED declines steeply with $\Gamma \sim 3.3$, and above $500 \mathrm{eV}$, the intrinsic photon index is $\Gamma \simeq 2.4$. As per Nardini et al. (2015), the total 1-1000 Rydberg ionizing luminosity is $5 \times 10^{46}-1 \times 10^{47} \mathrm{erg} \mathrm{s}^{-1}$, depending on the exact SED model adopted. Note that the main impact of using this SED form as the input photoionizing continuum is in the ionization balance of the gas; when compared to a model computed with a standard $\Gamma=2 \mathrm{X}$-ray power-law continuum, the ionization parameter will be comparatively higher as there are a larger number of input UV and soft X-ray ionizing photons in the SED form, resulting in a higher ionizing luminosity over the 1-1000 Rydberg band. This is particularly critical in the case of PDS 456, which has a steep UV to X-ray continuum; as a result, the ionization parameter can be an order of magnitude higher when compared to a model produced with a flat $\Gamma=2$ illuminating continuum.

Subsequently, a tabulated grid of XSTAR models was calculated by adopting this double broken power-law SED, with an input ionizing luminosity of $1 \times 10^{47} \mathrm{erg} \mathrm{s}^{-1}$ and an electron density of $n_{\mathrm{e}}=10^{8} \mathrm{~cm}^{-3}$, although the output spectra are largely insensitive to the density that is chosen. The grid of models also covers a wide range of parameter space, in the range $5 \times 10^{20}<N_{\mathrm{H}}<2.5 \times 10^{23} \mathrm{~cm}^{-2}$ in column density (in steps of $\left(\log N_{\mathrm{H}}\right)=0.14$ ) and in $2<\log \xi<7$ (in steps of $(\log \xi)=0.2)$ in terms of the ionization parameter. The subsequent output spectra were calculated over the $0.1-20 \mathrm{keV}$ range over 10,000 energy steps. A turbulence velocity of $\sigma_{\text {turb }}=5000 \mathrm{~km} \mathrm{~s}^{-1}$ was chosen for the final grid of models, as it was found that much lower turbulences could not match the breadth or equivalent width of the soft X-ray absorption features, while for higher turbulences, the absorption profiles became too broad to model the observed features seen near $1 \mathrm{keV}$ in the RGS spectra.

Given the good agreement between the RGS and MOS for the XMM-Newton sequences, for each sequence they were fitted simultaneously, allowing for a cross-normalization factor (typically within $\pm 5 \%$ of 1.0 ) between the two instruments. This has the benefit of utilizing both the high spectral resolution of the RGS and the higher $\mathrm{S} / \mathrm{N}$ of the MOS data. 
Table 3

Best-Fit Parameters of the Strongest Absorption Lines Detected in the MOS Spectra

\begin{tabular}{|c|c|c|c|c|c|c|}
\hline & $\begin{array}{l}\text { Rest Energy }{ }^{\mathrm{a}} \\
(\mathrm{eV})\end{array}$ & $\begin{array}{c}\text { Intensity } \\
\left(10^{-5} \mathrm{ph} \mathrm{cm}^{-2} \mathrm{~s}^{-1}\right)\end{array}$ & $\begin{array}{l}\text { EW } \\
(\mathrm{eV})\end{array}$ & $\begin{array}{c}\sigma \\
(\mathrm{eV})\end{array}$ & $\Delta \chi^{2} / \Delta \nu$ & $P_{\mathrm{F}}^{\mathrm{b}}$ \\
\hline \multicolumn{7}{|c|}{ OBS. B: No Gaussian lines, $\chi^{2} / \nu=172.3 / 101, N_{\mathrm{P}}=1.3 \times 10^{-5 \mathrm{c}}$} \\
\hline ABS2 & $1253_{-18}^{+18}$ & $-2.1_{-0.7}^{+0.7}$ & $-7.7_{-2.6}^{+2.5}$ & $100^{\mathrm{d}}$ & $34.1 / 2$ & $5.5 \times 10^{-6}$ \\
\hline \multicolumn{7}{|c|}{ OBS. CD: No Gaussian lines, $\chi^{2} / \nu=198.1 / 102, N_{\mathrm{P}}=3.8 \times 10^{-8 \mathrm{c}}$} \\
\hline \multicolumn{7}{|c|}{ OBS. E: No Gaussian lines, $\chi^{2} / \nu=208.3 / 101, N_{\mathrm{P}}=1.9 \times 10^{-9 c}$} \\
\hline ABS1 & $998_{-16}^{+17}$ & $-6.2_{-1.8}^{+2.1}$ & $-16.8_{-5.2}^{+5.7}$ & $45_{-15}^{+30}$ & $38.7 / 3$ & $5.8 \times 10^{-6}$ \\
\hline $\mathrm{ABS} 2$ & $1172_{-14}^{+13}$ & $-4.0_{-0.9}^{+0.9}$ & $-19.2_{-4.2}^{+4.4}$ & $45^{\mathrm{e}}$ & $33.4 / 2$ & $7.4 \times 10^{-6}$ \\
\hline EMIS1 & $933 \pm 23$ & $3.2_{-1.6}^{+1.7}$ & $7.1_{-3.0}^{+3.1}$ & $10^{\mathrm{d}}$ & $44.7 / 2$ & $2.4 \times 10^{-7}$ \\
\hline \multicolumn{7}{|c|}{ 2001: No Gaussian lines, $\chi^{2} / \nu=260.3 / 100, N_{\mathrm{P}}=3.16 \times 10^{-16 \mathrm{c}}$} \\
\hline $\mathrm{ABS} 2$ & $1254_{-13}^{+12}$ & $-8.6_{-1.6}^{+1.7}$ & $-22.9_{-4.2}^{+4.6}$ & $47^{\mathrm{e}}$ & $74.7 / 2$ & $1.1 \times 10^{-11}$ \\
\hline
\end{tabular}

Notes.

${ }^{a}$ Measured line energy in the PDS 456 rest frame.

${ }^{\mathrm{b}}$ Null-hypothesis probability of any individual line, via $F$-test.

${ }^{c}$ Fit statistic and null-hypothesis probability against the baseline continuum model (without Gaussian lines).

${ }^{\mathrm{d}}$ Denotes a fixed parameter. Where the line width is unresolved in the MOS, it is fixed at a width of $\sigma=10 \mathrm{eV}$. For the broad (ABS 1$)$ profile in OBS. CD, the width of $\sigma=110 \mathrm{eV}$ is fixed to the line width obtained from the RGS spectrum.

${ }^{\mathrm{e}}$ Denotes a width that is tied between the two pairs of absorption lines.

The spectra were fitted between 0.6 and $2.0 \mathrm{keV}$, avoiding the energy band below $0.6 \mathrm{keV}$ in order to minimize any calibration uncertainties associated with the $\mathrm{O}$ edge in both detectors. We did not directly fit the MOS spectra above $2 \mathrm{keV}$ as our motivation was to study the properties of the soft X-ray absorption. However, as a consistency check, we did extrapolate the spectra above $2 \mathrm{keV}$ to see whether, to first order, the model reproduced the continuum form above $2 \mathrm{keV}$. The continuum model adopted was the same as in the Gaussian fitting, that is, a power-law plus blackbody component absorbed by Galactic absorption. Generally, the photon index of the power law was fixed in all of the sequences to $\Gamma=2.1$, but in OBS. B and 2007 this was found to be steeper with $\Gamma=2.4-2.5$. In the 2001 sequence, which has a strongly absorbed spectrum, an additional neutral, partial covering absorption component (with $N_{\mathrm{H}}=4.8_{-0.9}^{+1.1} \times 10^{22} \mathrm{~cm}^{-2}$ and covering fraction $f_{\text {cov }}=0.34 \pm 0.02$ ) was applied to the soft $\mathrm{X}$-ray power law, because otherwise the photon index was found to be unusually hard with $\Gamma \sim 1$. We note that a similar partial covering component has been found in some of the previous Suzaku observations of PDS 456, when the spectrum becomes unusually hard (Gofford et al. 2014; Reeves et al. 2014; Matzeu et al. 2016).

\subsection{Fitting Methodology}

The XSTAR absorption grids were then applied to each of the sequences, allowing the column density, ionization parameter, and outflow velocity to vary independently, as well as the continuum parameters. In order to find the best-fit solution for each absorption zone as fitted to each sequence in velocity space, we first varied the outflow velocity from $-0.3 c$ (where the negative sign here denotes blueshift) up to $+0.3 c$, in steps of $0.005 c$, while allowing the ionization and column to adjust at each step in the fitting. Thus no a priori assumption is made about the likely velocity of any of the absorbers fitted to each sequence, and the wide range in velocity space searched ensures that the global minimum is found, allowing for any possible zero-velocity solution as well as outflow or inflow. This procedure was then repeated for each absorption zone that was subsequently added to each sequence, applying the criteria that the addition of each absorption grid to the model required an improvement in fit statistic corresponding to at least the 99.9\% confidence level. Once the global minimum for each absorption grid was found, we then conducted a finer search in velocity, ionization, and column density in order to find the exact minimum and uncertainties associated with each parameter.

As an example, we describe in detail how this was applied to the OBS. E spectra, while the same fitting procedure was applied to all of the sequences. The initial starting point was with the baseline continuum model, with only the Galactic absorption component present (and no intrinsic absorption), which yielded a poor fit statistic of $\chi^{2} / \nu=289.0 / 161$. A single ionized zone of absorption was then added to the model, and the $\chi^{2}$ space was searched by stepping through the fit at each point in the outflow velocity, as described above. Figure 8 (upper panel) shows the resulting fit statistic against outflow velocity for this absorber. A clear minimum is found at $v=-0.17 \pm 0.01 c$, while a zero-velocity solution is ruled out at $>99.99 \%$ confidence. As a result of the addition of this single absorber with an outflow velocity of $-0.17 c$, the fit statistic improved to $\chi^{2} / \nu=206 / 158$. The corresponding $F$-test null hypothesis upon adding the first zone of absorption to the model (compared to the model with no absorption) is 

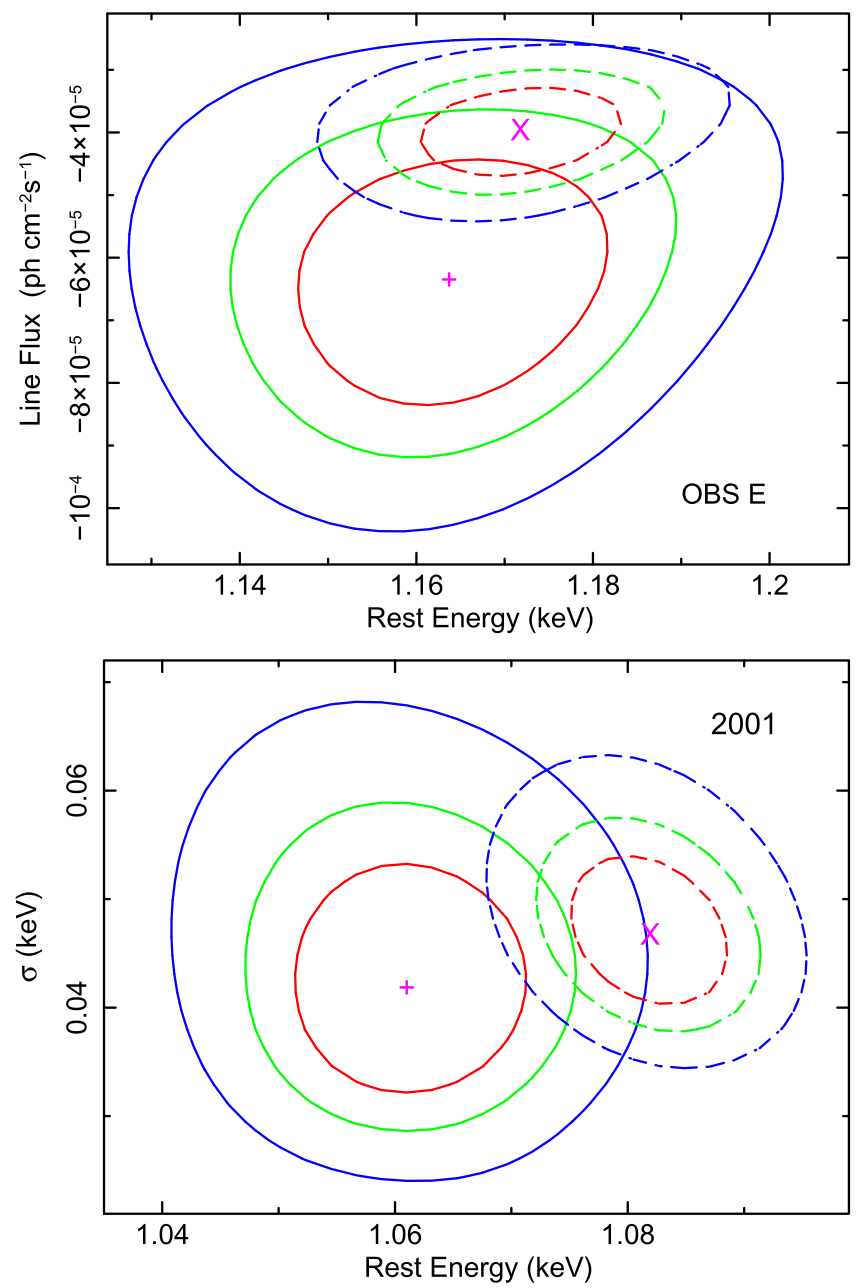

Figure 7. Contour plots, showing the comparison between two of the broad absorption lines detected in the RGS and MOS spectra. The contours for the RGS are shown as solid lines, while the MOS is indicated by dot-dashed lines. The contours represent the $68 \%, 90 \%$, and $99 \%$ levels for two parameters of interest. The upper panel shows the contours between rest energy and line flux for the $1.16 \mathrm{keV}$ absorption line in the OBS. E spectrum, illustrating the independent agreement between the two spectra. The lower panel shows the contours for the broad absorption trough in the 2001 spectrum, showing the agreement in the width of the line profile $(\sigma)$ measured between the two detectors.

very low, with $P_{\mathrm{f}}=1.3 \times 10^{-11}$. A second absorption grid was then added to the model, to test whether the fit improved further upon its addition, and the velocity space of the new absorber was searched for any minimum in $\chi^{2}$. The result of adding this second absorption grid to the model is shown in Figure 8 (lower panel), which also shows a well-defined minimum, but at a lower outflow velocity of $v=-0.06 \pm 0.01 c$. The fit statistic also significantly improved upon the addition of the second absorption grid, with a resulting reduced chi-squared of $\chi^{2} / \nu=181 / 155$. The corresponding $F$-test null hypothesis upon adding the second zone of absorption to the model (compared to the model with only one absorber) is then $P_{\mathrm{f}}=1.6 \times 10^{-4}$. The addition of further absorption grids to the OBS. E spectra did not significantly improve the fit.

Thus the OBS. E spectrum requires two absorption zones, one with a faster outflow velocity $(-0.17 c)$, while both zones formally require an outflow velocity at $>99.9 \%$ confidence. The resulting best-fit absorption parameters for both OBS. E as
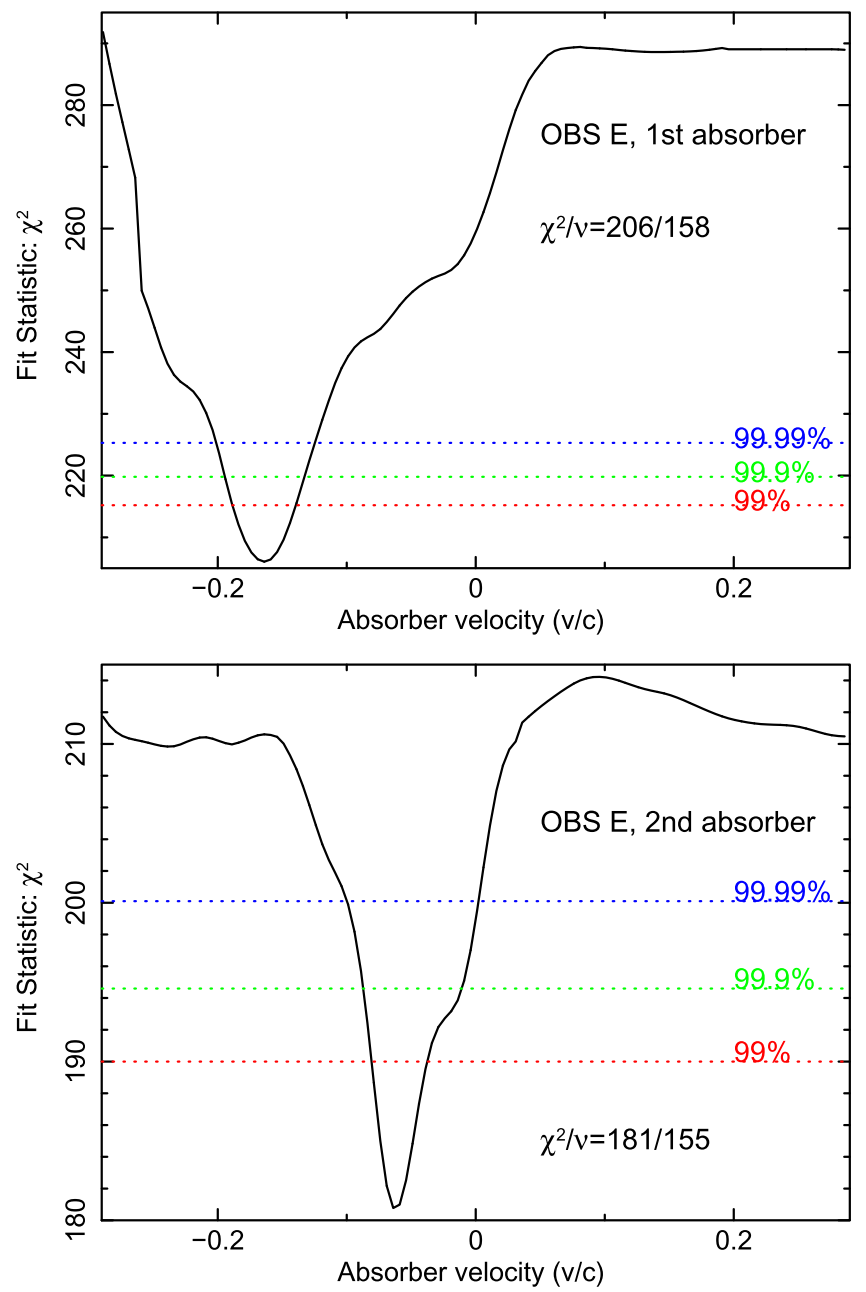

Figure 8. Fit minimization in terms of $\chi^{2}$ vs. velocity for the OBS. E RGS + MOS spectra. Here a negative velocity represents outflow or blueshift. The plots show the changes in the resultant $\chi^{2}$ after stepping through velocity space for the addition of two successive absorption zones to the model, as described in the text. The upper panel shows a well-determined minimum at an outflow velocity of $v_{\text {out }}=-0.17 c$ for the fast absorption zone. After accounting for the fast zone in the fit, the lower panel shows a further minimum of $v_{\text {out }}=-0.06 c$ corresponding to an additional slower absorption zone. The horizontal dotted lines correspond to the $99 \%, 99.9 \%$, and $99.99 \%$ confidence intervals for the two parameters of interest. Note that a solution with zero velocity or redshift is clearly excluded by the data. The resultant best-fit reduced chi-squared upon the addition of each absorption zone is also shown in each panel.

well as the other spectral sequences are shown in Table 4, after applying the same fitting procedure as described above. In general, the soft X-ray absorption can primarily be modeled with two main XSTAR zones (labeled as zones 1 and 2, respectively, in Table 4), both of which are required to be outflowing with respect to the systemic velocity of PDS 456, while we refer to zone 2 as having the higher velocity of the two zones.

\subsection{Results of Photoionization Modeling to OBS. E}

\subsubsection{A Self-consistent Emission and Absorption Model}

In addition to the two absorption zones described above, the fit to OBS. E improves further to $\chi^{2} / \nu=143.2 / 150$ by adding two broad Gaussian emission profiles. The emission-line centroids are at $922 \pm 9 \mathrm{eV}$ and $1066 \pm 16 \mathrm{eV}$ and are apparent in the MOS and RGS spectra for OBS. E (see 
Table 4

Best-fit Parameters for the Ionized Absorbers

\begin{tabular}{|c|c|c|c|c|c|c|}
\hline & & B & $\mathrm{CD}$ & $\bar{E}$ & 2001 & 2007 \\
\hline \multirow[t]{3}{*}{ Zone 1} & $N_{\mathrm{H}}{ }^{\mathrm{a}}$ & $\ldots$ & $\ldots$ & $0.61_{-0.20}^{+0.25}$ & $1.9_{-0.5}^{+0.5}$ & $\ldots$ \\
\hline & $\log \xi^{\mathrm{b}}$ & $\ldots$ & $\ldots$ & $3.65_{-0.13}^{+0.13}$ & $4.09_{-0.10}^{+0.07}$ & $\cdots$ \\
\hline & $v_{\text {out }} / c$ & $\ldots$ & $\ldots$ & $-0.064_{-0.012}^{+0.012}$ & $-0.082_{-0.01}^{+0.01}$ & $\ldots$ \\
\hline \multirow[t]{3}{*}{ Zone 2} & $N_{\mathrm{H}}^{\mathrm{a}}$ & $0.52_{-0.20}^{+0.29}$ & $0.70_{-0.21}^{+0.22}$ & $1.50_{-0.45}^{+0.40}$ & $2.2_{-0.6}^{+0.7}$ & $<0.23$ \\
\hline & $\log \xi^{\mathrm{b}}$ & $4.04_{-0.29}^{+0.13}$ & $4.18_{-0.08}^{+0.05}$ & $4.18_{-0.11}^{+0.08}$ & $4.19_{-0.08}^{+0.07}$ & $4.10^{f}$ \\
\hline & $v_{\text {out }} / c$ & $-0.254_{-0.013}^{+0.011}$ & $-0.267_{-0.007}^{+0.006}$ & $-0.170_{-0.011}^{+0.012}$ & $-0.237_{-0.013}^{+0.013}$ & $-0.177_{-0.011}^{+0.015}$ \\
\hline \multirow[t]{4}{*}{ Zone 3} & $N_{\mathrm{H}}{ }^{\mathrm{a}}$ & $<31$ & $23_{-8}^{+8}$ & $<37$ & $<46$ & $<22$ \\
\hline & $\log \xi^{\mathrm{b}}$ & $5.5^{\mathrm{c}}$ & $5.5^{\mathrm{c}}$ & $5.5^{\mathrm{c}}$ & $5.5^{\mathrm{c}}$ & $5.5^{\mathrm{c}}$ \\
\hline & $v_{\text {out }} / c$ & $-0.3^{\mathrm{c}}$ & $-0.30_{-0.01}^{+0.01}$ & $-0.3^{\mathrm{c}}$ & $-0.3^{\mathrm{c}}$ & $-0.3^{\mathrm{c}}$ \\
\hline & $\Delta \chi^{2}$ & $\ldots$ & 20.0 & $\cdots$ & $\ldots$ & 10.9 \\
\hline
\end{tabular}

Notes.

${ }^{\text {a }}$ Column density in units of $10^{22} \mathrm{~cm}^{-2}$. Note that for OBS. A, the upper limit on the column density (EPIC only) is $<3.5 \times 10^{21} \mathrm{~cm}^{-2}$ for an ionization parameter of $\log \xi=4.1$.

$\mathrm{b}$ Ionization parameter in units of $\mathrm{erg}_{\mathrm{cm} \mathrm{s}}{ }^{-1}$.

${ }^{\mathrm{c}}$ Denotes a fixed parameter.
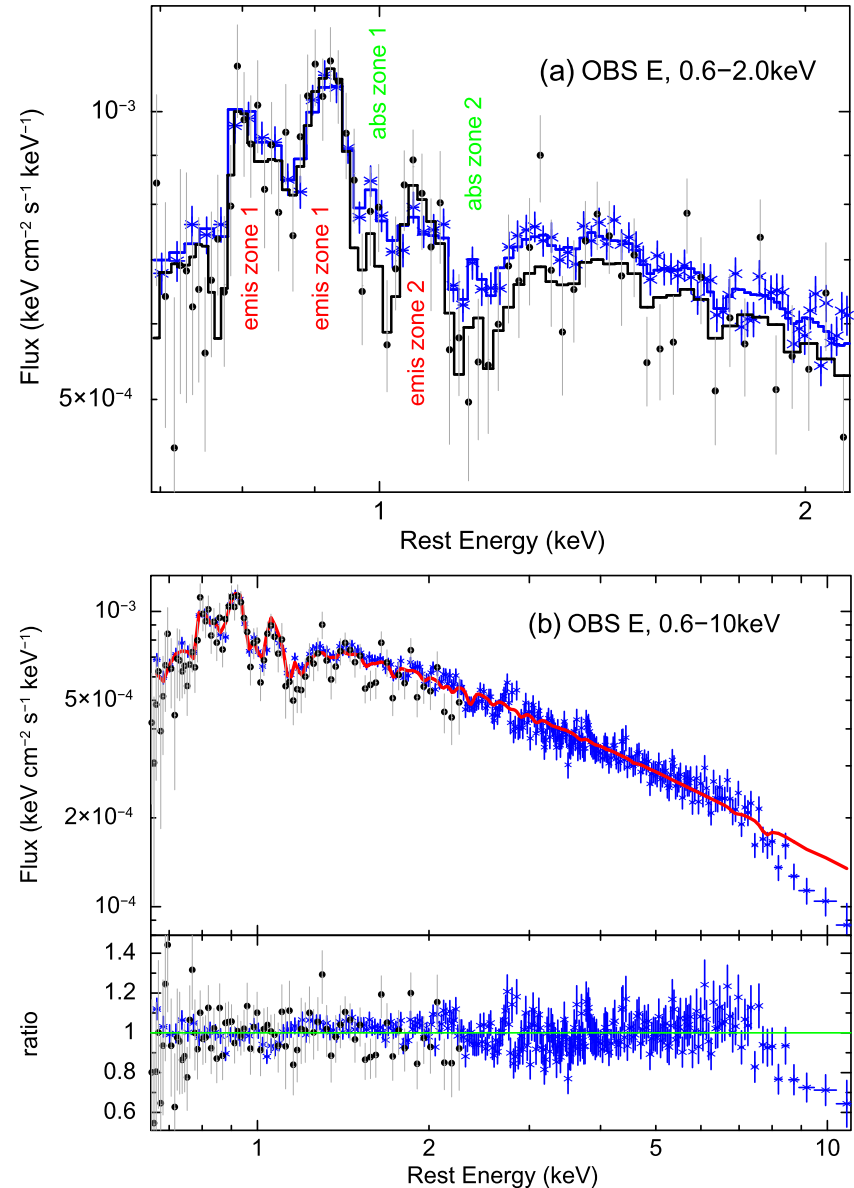

Figure 9. Best-fit XSTAR absorption model applied to observation E. The top panel shows the XSTAR model fitted simultaneously from 0.6 to $2.0 \mathrm{keV}$ to the MOS (data points, blue stars) and RGS (black circles), while the best-fit model fitted to each instrument is a solid line. The XSTAR model is able to account for the absorption structure with two different velocity zones of absorbing gas (absorption zones 1 and 2 in Table 4) and the excess emission with two corresponding zones (see Table 5). The lower panel shows the same model, but extrapolated up to $10 \mathrm{keV}$ in the MOS. The soft X-ray absorption model is able to account for the broadband spectrum up to $10 \mathrm{keV}$, with the exception of the P-Cygni-like absorption feature at iron K.
Table 5

Photoionized Emission Parameters for Observation E

\begin{tabular}{lcc}
\hline \hline Parameter & Zone 1 (Low Ionization) & Zone 2 (High Ionization) \\
\hline$N_{\mathrm{H}}{ }^{\mathrm{a}}$ & $0.6^{\mathrm{b}}$ & $1.5^{\mathrm{b}}$ \\
$\log \xi^{\mathrm{c}}$ & $2.8 \pm 0.3$ & $4.6_{-0.4}^{+0.6}$ \\
$\nu_{\text {out }} / c$ & $-0.040 \pm 0.018$ & $-0.08 \pm 0.02$ \\
$\kappa_{\mathrm{xstar}}{ }^{\mathrm{C}}$ & $6.0 \pm 3.0 \times 10^{-4}$ & $<1.2 \times 10^{-3}$ \\
$f=\Omega / 4 \pi^{\mathrm{e}}$ & $0.44 \pm 0.22$ & $<0.88$ \\
\hline
\end{tabular}

Notes.

${ }^{\text {a }}$ Column density in units of $10^{22} \mathrm{~cm}^{-2}$. Note the column is tied to the respective absorption zone.

${ }^{\mathrm{b}}$ Denotes a fixed parameter.

${ }^{c}$ Ionization parameter in units of $\mathrm{erg}_{\mathrm{cm} \mathrm{s}}{ }^{-1}$.

${ }^{\mathrm{d}}$ The normalization of the XSTAR component, which is defined as $\kappa=f L_{38} / D_{\mathrm{kpc}}^{2}$, where $L_{38}$ is the ionizing luminosity in units of $10^{38} \mathrm{erg} \mathrm{s}^{-1}$, $D_{\mathrm{kpc}}$ is the source distance in kpc, and $f$ is the covering fraction of the photoionized gas. See Section 6.2 for details.

${ }^{\mathrm{e}}$ Covering fraction, where $f=\Omega / 4 \pi$.

Figure 9), while the lines have fluxes of $7.5_{-2.9}^{+4.0} \times 10^{-5}$ and $3.2_{-1.8}^{+2.2} \times 10^{-5}$ photons $\mathrm{cm}^{-2} \mathrm{~s}^{-1}$, respectively (or equivalent widths of $17 \pm 8$ and $11 \pm 7 \mathrm{eV}$ ). Note that strong $0.92 \mathrm{keV}$ line emission has also been detected previously in some of the Suzaku X-ray Imaging Spectrometer (XIS) spectra of PDS 456; see, for instance, Figure 2 in Reeves et al. (2009). The line width, assuming a common width between the lines, is $\sigma=20 \pm 8 \mathrm{eV}$ (or equivalently, $\sigma_{\mathrm{v}}=6500 \pm 2600 \mathrm{~km} \mathrm{~s}^{-1}$ ).

In order to model the emission, we removed the ad hoc Gaussian emission lines from the model and instead fitted the emission using a self-consistent grid of photoionized emission model spectra produced by XSTAR, with the same input parameters and turbulence velocity $\left(\sigma_{\text {turb }}=5000 \mathrm{~km} \mathrm{~s}^{-1}\right)$ as per the absorption grids. Like for the absorption modeling, two emission grids were added to the model, with the column densities of the two emission zones tied to the corresponding values in the two absorption zones. The ionization, outflow velocity, and normalization (which is proportional to luminosity) of the two emission grids were allowed to vary. The 
parameters of the two emission zones are reported in Table 5 as applied to OBS. E. The most significant emission zone is zone 1, which is also the lower ionization of the two zones $(\log \xi=2.8 \pm 0.3)$ and yields an improvement in fit statistic of $\Delta \chi^{2} / \Delta \nu=23.0 / 3$ upon its addition to the baseline absorption model, while in comparison the second and more highly ionized zone $(\log \xi \sim 4.6)$ is only marginally significant $\left(\Delta \chi^{2} / \Delta \nu=12.0 / 3\right)$.

This final XSTAR model provides a good fit to the simultaneous RGS and MOS spectra in OBS. E, with an overall fit statistic of $\chi^{2} / \nu=146.5 / 149$. The resulting fit is shown in Figure 9, panel (a), with the XSTAR model able to reproduce the absorption features, that is, the two broad absorption troughs at rest-frame energies of $\sim 1.0$ and $\sim 1.2 \mathrm{keV}$. While the emitters do not necessarily have to be physically associated with the corresponding absorption zones, the emission is nonetheless able to reproduce the excess soft $\mathrm{X}$-ray emission. In particular, the lower ionization emission zone 1 accounts for the stronger emission line observed near $920 \mathrm{eV}$. Emission zone 1 also predicts an emission feature near $0.8 \mathrm{keV}$, which is consistent with the spectrum shown in Figure 9. Emission zone 2 has a more subtle effect, mainly accounting for the weaker emission line near $1.06 \mathrm{keV}$. If this best-fit model to the soft X-ray spectrum of OBS. $\mathrm{E}$ is extrapolated up to higher energies using the MOS data, the agreement is good, with the model reproducing well the shape and level of the continuum above $2 \mathrm{keV}$, as is seen in Figure 9, panel (b). The remaining residuals at high energies are due to the iron $\mathrm{K}$ absorption profile, as reported in Nardini et al. (2015).

\subsubsection{Properties of the Soft X-ray Emission and Absorption}

In terms of the absorber properties, zone 1 has a lower column and lower ionization compared to zone 2 (see Table 4 for parameter details), as well as a lower outflow velocity of $v_{\text {out }} / c=-0.064 \pm 0.012 \quad$ (compared to $\quad v_{\text {out }} / c=-0.17$ \pm 0.01 in zone 2), and primarily accounts for the lowest energy of the two absorption troughs. Note that a nonzero outflow velocity is required for both zones in the fit, as is also described in the fitting procedure described in Section 5.2 (see Figure 8). With the emitter included, if the velocity of both zones is forced to zero and the model refitted allowing the other absorber parameters to vary $\left(N_{\mathrm{H}}, \log \xi\right)$, then the fit is substantially worse than in the case where the outflow velocities are allowed to vary $\left(\chi^{2} / \nu=181.2 / 152\right.$ versus $\left.\chi^{2} / \nu=143.2 / 150\right)$. On the other hand, if we do allow the velocity to vary, but assume that it is the same for both zones, then the fit statistic is also worse than before $\left(\chi^{2} / \nu=176.4 / 151\right)$, because adjusting the ionization alone is not enough to explain the different energy centroids of the absorption profiles. Thus the two absorber zones are required to be distinct in velocity space, as well as in column and ionization.

In Figure 10 the relative contribution of each absorption zone is shown in terms of the fraction of the continuum that is absorbed against energy. The main contribution to zone 1 arises from $\mathrm{Ne} I \mathrm{X}-\mathrm{X}$, as well as absorption from L-shell Fe from ions in the range $\mathrm{Fe} \mathrm{XIX}-\mathrm{Xx}$; the blend of these lines together with a modest net blueshift produces the overall broad absorption trough that is centered near $1 \mathrm{keV}$ in the rest frame. The contribution to zone 2 is similar, giving a blend of lines from $\mathrm{Ne} I \mathrm{X}-\mathrm{X}$ and Fe XX-XIV; then the higher energy of this

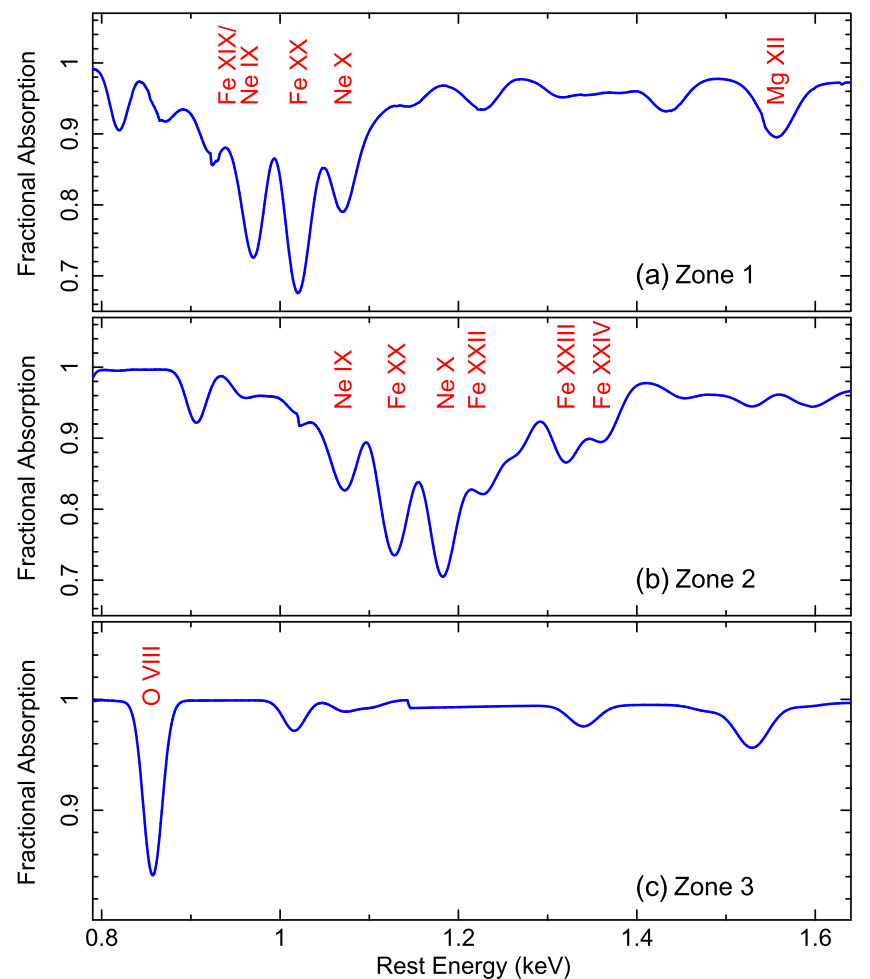

Figure 10. A graphical representation of the three XSTAR absorber zones (see Table 4), showing the fraction of the continuum absorbed by each zone in the region around $1 \mathrm{keV}$. Zone 1 (upper panel) corresponds to the lowest velocity $(0.06 c)$, lower ionization component in OBS. E, which mainly produces the $1 \mathrm{keV}$ absorption trough seen in this observation. Zone 2 (middle panel) is a faster zone, and the blend of lines is responsible for the broad absorption trough observed near $1.2 \mathrm{keV}$. Most of the contribution to zones 1 and 2 arises from the L-shell lines of iron and K-shell Ne. Zone 3 (lower panel) is the highest ionization and fastest zone present in OBS. CD, which makes only a small contribution to the soft band, as it is mainly responsible for the blueshifted absorption near $0.8 \mathrm{keV}$, which is due to $\mathrm{O}$ VIII Ly $\alpha$.

absorption blend (centered near $1.2 \mathrm{keV}$ ) arises primarily from the higher outflow velocity and somewhat from the higher ionization of this zone.

Note that although either the 1 or $1.2 \mathrm{keV}$ absorption troughs in OBS. E could at first sight be associated with absorption from $\mathrm{Ne}$ x (at $1.02 \mathrm{keV}$ ) or Fe XXIV (at $1.16 \mathrm{keV}$ ) with a smaller blueshift, the self-consistent XSTAR modeling presented above appears to rule this out. This is because it is difficult to produce absorption from these species in isolation, without a contribution from other, lower-energy, lines. For instance, for the higher-velocity zone, although the Fe XXIV $2 s \rightarrow 3 p$ absorption can contribute to the broad absorption profile at $1.2 \mathrm{keV}$, the other lower-energy L-shell Fe lines near $1 \mathrm{keV}$ also strongly contribute, reducing the overall centroid energy of the absorption trough produced in the XSTAR model (see Figure 10 for their relative contributions). The net result is that an overall blueshift of the absorption profile is required to match its observed energy in the actual spectrum.

In terms of the emission, the strongest contribution is from zone 1, which has the lowest ionization parameter. The outflow velocities of the two emission zones are lower than their respective absorption zones; that is, the outflow velocity of zone 1 is $v_{\text {out }}=-0.04 \pm 0.02 c$. This could be consistent with the emission expected from a biconical outflow, as emission can be observed across different sightlines that will appear to have a lower net outflow velocity than along the direct line of 


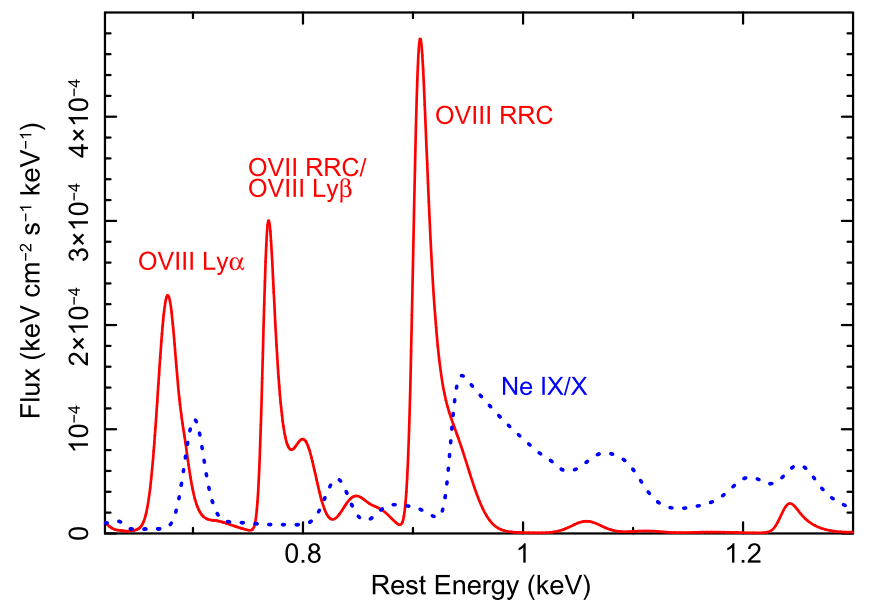

Figure 11. The relative contribution of the two soft X-ray photoionized emission zones from XMM-Newton OBS. E, as modeled by XSTAR with a velocity broadening of $\sigma=5000 \mathrm{~km} \mathrm{~s}^{-1}$ (see Table 5). The solid (red) line represents the lower-ionization $(\log \xi=2.8)$ emission zone 1 , which contributes to the majority of the soft X-ray line emission. In particular, the strongest line observed at $\sim 0.9 \mathrm{keV}$ likely originates from the blueshifted $\mathrm{O}$ VIII $\mathrm{RRC}$, while at lower energies the $\mathrm{O}$ VIII Ly $\alpha$ line is comparatively weaker as it is suppressed by Galactic absorption. The dashed (blue) line illustrates the much weaker contribution that the higher-ionization zone 2 makes to the emission spectrum.

sight to the observer. The emission from the near side of the outflow may be preferentially observed in this case if the emission from the far (receding) side of the wind is obscured through a greater column of matter. This may be the case if, for instance, the receding wind is on the far side of the accretion disk.

The relative contribution of the photoionized emission zones to the soft X-ray spectrum of PDS 456 are shown in Figure 11. The dominant contribution to the emission is from zone 1 (Figure 11, red curve), and the majority of the emission arises from $\mathrm{O}$ VII and $\mathrm{O}$ VIII. In particular, the emission near $0.9 \mathrm{keV}$ is mainly accounted for by the blueshifted $\mathrm{O}$ VIII radiative recombination continuum (RRC) at $0.87 \mathrm{keV}$, along with some contribution from the forbidden line of Ne IX. At first sight, the $\mathrm{O}$ VIII RRC appears stronger than the $\mathrm{O}$ VIII Ly $\alpha$ line at lower energies. However, this is just due to the latter emission being more suppressed by the Galactic absorption toward PDS 456, as this lower-energy line falls just above the deep neutral $\mathrm{O}_{\mathrm{I}}$ edge, as seen in the observed frame. In contrast, the higherionization $\left(\log \xi=4.6_{-0.4}^{+0.6}\right.$ ) zone 2 is much weaker (Figure 11, blue curve), and its main contribution is to reinforce the emission near $1 \mathrm{keV}$, mainly due to blueshifted emission from $\mathrm{Ne}$ IX and $\mathrm{Ne} \mathrm{X}$.

\subsection{Extension to Other XMM-Newton Observations}

The absorption modeling was then extended to the other 2013-2014 sequences, as well as the 2001 and 2007 XMMNewton observations. In these observations, the soft X-ray emission is less prominent because the continuum fluxes are generally higher than in OBS. E, so we subsequently concentrated on modeling only the absorption zones. The zone 2 absorber appears common to all of the data sets, except for the bright and featureless 2007 spectrum, where only an upper limit can be placed on the column. Indeed, the ionization parameter of this zone is consistent within errors for all the data sets, varying only in the narrow range of $\log \xi=4.04-4.19$ (see Table 4). The main differences arise in the column density, which is higher in the moreabsorbed OBS. E $\left(N_{\mathrm{H}}=1.5 \pm 0.4 \times 10^{22} \mathrm{~cm}^{-2}\right)$ and 2001 $\left(N_{\mathrm{H}}=2.2_{-0.6}^{+0.7} \times 10^{22} \mathrm{~cm}^{-2}\right)$ sequences and weakest in the least-absorbed OBS. B and 2007 observations. Indeed in the bright, continuum-dominated 2007 observations, a low upper limit can be placed on the column density of $N_{\mathrm{H}}<0.23 \times 10^{22} \mathrm{~cm}^{-2}$. Likewise, although the RGS data are not available for OBS. A, on the basis of the EPIC/MOS data we can still place an upper limit on the column density of $N_{\mathrm{H}}<0.35 \times 10^{22} \mathrm{~cm}^{-2}$ for zone 2 , consistent with the absorption being weaker while the continuum flux was brighter during this observation.

The outflow velocity is strongly variable between some of sequences, such as $v_{\text {out }} / c=-0.27 \pm 0.01$ in OBS. CD versus $v_{\text {out }} / c=-0.17 \pm 0.01$ in OBS. E. This is illustrated in Figure 12, which shows the confidence contours in the $N_{\mathrm{H}}$ versus outflow velocity plane for zone 2 in each of the $\mathrm{B}, \mathrm{CD}$, $\mathrm{E}$, and 2001 sequences, the best-fit parameters clearly differing along the velocity axis. Thus, while the column determines the depths of the broad absorption troughs, the outflow velocity determines its blueshift. Indeed, in the CD observation it can be seen (e.g., from Figure 6) that the broad absorption trough is shallower and shifted to higher energies compared to observation E.

On the other hand, while the lower-velocity zone 1 is required to reproduce the lower-energy absorption troughs in OBS. E and 2001 near $1 \mathrm{keV}$, it is not required in any of the other observations and thus appears rather sporadic. Zone 3 is included for completeness and represents a possible highionization phase of the wind, similar to what is seen at iron $\mathrm{K}$, with a similar ionization level of $\log \xi=5.5$ and a velocity of $-0.3 c$ (Nardini et al. 2015). At this level of ionization, in the soft X-ray band, there is only a small imprint from zone 3 in the spectrum, which is mainly in the form of a $\mathrm{O}$ vIII Ly $\alpha$ absorption line blueshifted to above $0.8 \mathrm{keV}$ (see the lower panel of Figure 10), while only trace amounts of absorption due to $\mathrm{H}$-like $\mathrm{Ne}$ and $\mathrm{Mg}$ are predicted. Because of its transparency at soft X-rays, this highest-ionization zone is therefore only formally required in the OBS. CD spectrum, mainly to reproduce the weak absorption feature detected near $0.84 \mathrm{keV}$ in the RGS and MOS data (see Tables 2 and 3).

\section{DISCUSSION}

\subsection{The Detection of Fast Soft X-Ray Absorbers}

Although the numbers of confirmed fast outflows have significantly increased through systematic studies of AGNs in the iron K band (Tombesi et al. 2010; Gofford et al. 2013), to date there have been relatively few detections of fast outflows in the soft X-ray band. Here, the detection of broad soft X-ray absorption-line profiles associated with the fast wind in PDS 456 represents one of the few cases where the presence of an ultrafast outflow has been established in the form of highly blueshifted absorption in both the Fe K and soft X-ray bands. Other notable recent examples include the nearby QSO, PG 1211+143 (Pounds 2014), and, in the NLS1, IRAS 17020 +4544 (Longinotti et al. 2015). In both of these cases, the observations suggest that the outflow is more complex than a single-zone medium, with multiple ionization or velocity components. In PG $1211+143$, at least two different velocity components are present, both in the iron $\mathrm{K}$ band as well as soft 

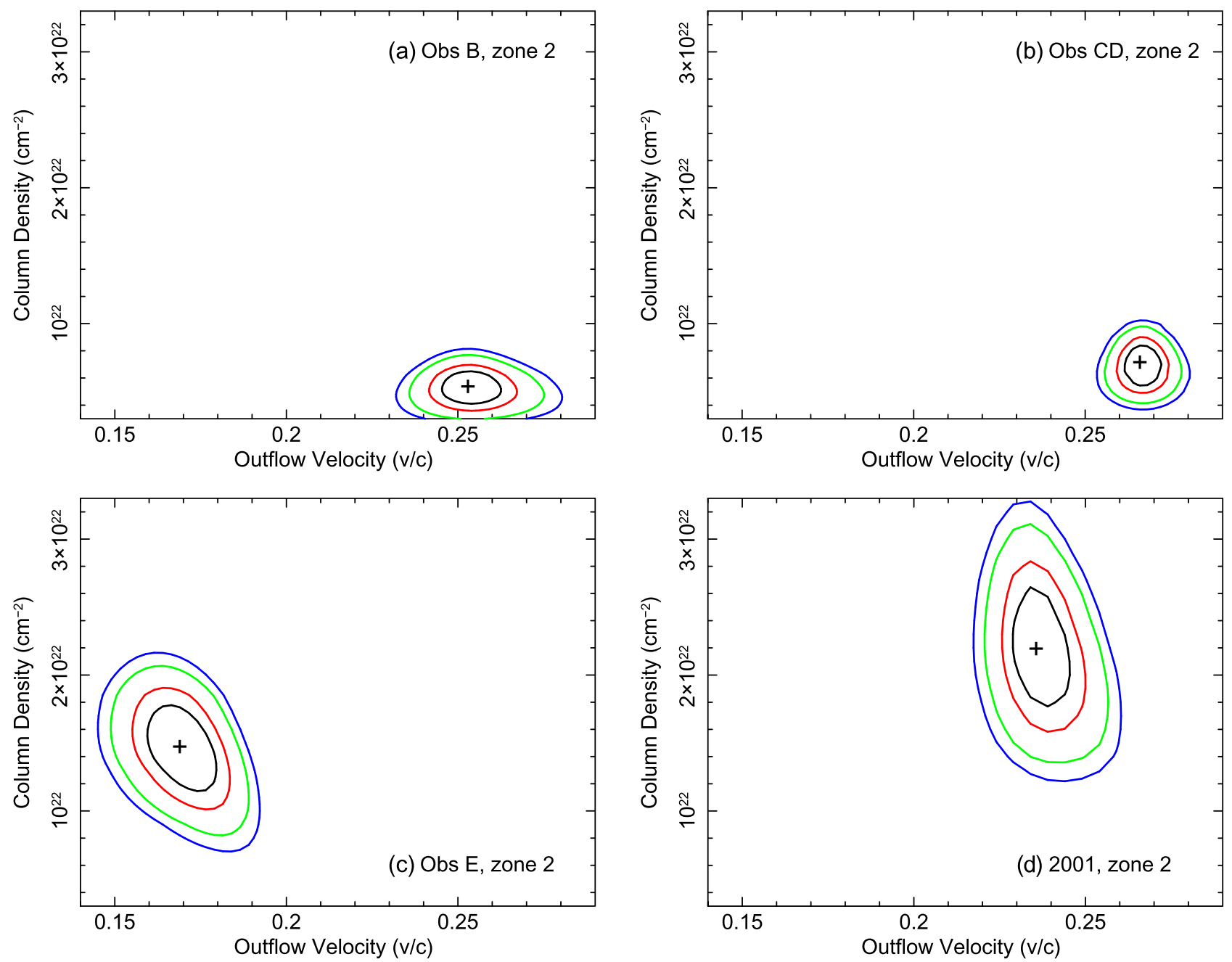

Figure 12. Contour plots of column density against outflow velocity for the zone 2 absorber for (a) OBS. B, (b) OBS. CD, (c) OBS. E, and (d) 2001. The inner (black) contour to the outermost (blue) contour represent the 68\%,90\%,99\%, and 99.9\% confidence contours for the two parameters of interest, while the black crosses represent the best-fit values. For OBS. B and OBS. CD, the confidence contours overlap, indicating that the soft X-ray absorber has not strongly varied on the 2 week timescale between these consecutive observations. However, on longer (months to years) timescales, the absorber clearly varies; for example, between OBS. E (2014 February) and OBS. CD (2013 September), the column has increased by at least a factor of 2, while the velocity has decreased from $0.27 c$ to $0.17 c$. Across all of the observations, the absorber varies in both $N_{\mathrm{H}}$ and velocity, while the ionization parameter remains constant within errors. Note the identical $y$ and $x$ axis scales on all four plots.

X-rays (Pounds et al. 2016), while in the NLS1 IRAS 17020 +4544 , a complex fast $\left(v_{\text {out }}=23,000-33,000 \mathrm{~km} \mathrm{~s}^{-1}\right)$ soft $\mathrm{X}$-ray absorber is present, covering at least three zones in terms of ionization parameter.

In PDS 456, while the velocity of the soft X-ray absorber (with $v_{\text {out }}=0.17-0.27 c$ for zone 2 ) is similar to the highly ionized iron $\mathrm{K}$ absorption in this $\mathrm{AGN}\left(v_{\text {out }}=0.25-0.3 c\right)$, the column density and ionization are up to two orders of magnitude lower than the iron $\mathrm{K}$ absorber, that is, $\log \xi \sim 4$ and $N_{\mathrm{H}}=10^{22} \mathrm{~cm}^{-2}$ at soft X-rays versus $\log \xi \sim 6$ and $N_{\mathrm{H}}=10^{24} \mathrm{~cm}^{-2}$ at iron $\mathrm{K}$. This also suggests that the outflow is more complex than what would be expected for a simple homogeneous radial outflow, where the density varies with radius as $n \propto r^{-2}$. Thus, as $\xi=L / n r^{2}$, in the radial case the ionization should remain approximately constant along the outflow. A density profile with a power-law distribution flatter than $n(r) \propto r^{-2}$ could account for a decrease in ionization with absorber radial distance, and indeed it has been suggested from the distribution of warm absorbing gas among several Seyfert 1's (Behar 2009; Tombesi et al. 2013). Another likely possibility, especially given the rapid variability of the soft $\mathrm{X}$-ray absorption seen here, is a clumpy, multiphase wind. Thus, denser (and more compact) clumps or filaments could coexist within a smoother, highly ionized outflow to explain the ionization gradients. Indeed, a recent, albeit slower $(v \sim 0.01 c)$, multiphase disk wind was recently revealed in the changing-look Seyfert galaxy, NGC 1365 (Braito et al. 2014), with very rapid changes in soft X-ray absorption (with $\Delta N_{\mathrm{H}} \sim 10^{23} \mathrm{~cm}^{-2}$ in $\Delta t<100 \mathrm{ks}$ ), requiring a clumpy outflowing medium.

\subsection{The Soft X-Ray Outflow}

We now explore the properties of the soft X-ray absorber within the context of a clumpy wind model. In order to place a radial constraint on the absorbing gas, its variability timescale is considered. We concentrate on zone 2, which is the best determined of the absorption zones and is relatively constant in ionization (see Table 4). During the first four observations in the 2013-2014 campaign, there is a subtle variation in the column density from $<0.35 \times 10^{22} \mathrm{~cm}^{-2}$ (OBS. A) to 
$0.70_{-0.21}^{+0.22} \times 10^{22} \mathrm{~cm}^{-2}$ (OBS. CD), that is, a slight increase over a 3 week timescale. A stronger variation occurs over the subsequent 5 month period between OBS. A-D and OBS. E, with an increase in column density to $1.5 \pm 0.4 \times 10^{22} \mathrm{~cm}^{-2}$, while the velocity of the absorber also decreases from $0.27 \pm 0.01 c$ to $0.17 \pm 0.01 c$. The more subtle absorption variability between OBS. A and OBS. D in 2013 likely corresponds to the passage of the same absorbing system (with the same outflow velocity) across the line of sight. However, the stronger variations in $N_{\mathrm{H}}$ and outflow velocity between this and OBS. E (as well as in 2001) likely correspond to different absorption systems (with 2007 being unabsorbed). Therefore, we set a plausible timescale for the absorption variability, in terms of the passage of clumps or filaments of gas across our line of sight, of $\Delta t=10^{6}-10^{7} \mathrm{~s}$, that is, weeks to months. It is also likely that some variability can occur on shorter timescales, as found during the low-flux 2013 Suzaku observations, where changes in the soft X-ray spectrum on timescales of $\gtrsim 100$ ks could be accounted for by variability of a partially covering absorber (Matzeu et al. 2016).

The transverse motion of the absorbing systems moving across the line of sight is assumed to be due to the overall Keplerian rotation of the gas within the wind, with the observed outflow velocity being due to the bulk motion of the wind toward us. The Keplerian velocity at a radius $R$ is simply $v_{\mathrm{K}}^{2}=c^{2} / r_{\mathrm{g}}$, where $r_{\mathrm{g}}=R / R_{\mathrm{g}}$ is the radial distance from the black hole in gravitational units. The column through an (approximately spherical) absorbing cloud can be expressed as $N_{\mathrm{H}} \sim n_{\mathrm{H}} \Delta R$, where its size is $\Delta R=v_{\mathrm{K}} \Delta t$. The number density of an absorbing cloud is then given as

$$
n_{\mathrm{H}} \sim \frac{N_{\mathrm{H}}}{\Delta R}=\frac{N_{\mathrm{H}} r_{\mathrm{g}}^{1 / 2}}{c \Delta t} .
$$

The definition of the ionization parameter gives $n_{\mathrm{H}} \sim n_{\mathrm{e}}=L_{\text {ion }} / \xi R^{2}$, so equating these densities yields the radial distance of the clouds:

$$
r_{\mathrm{g}}^{5 / 2}=\frac{L_{\mathrm{ion}}}{\xi} \frac{c^{5} \Delta t}{N_{\mathrm{H}}}\left(G M_{\mathrm{BH}}\right)^{-2} .
$$

For the zone 2 absorber, $\log \xi \sim 4$ and $N_{\mathrm{H}} \sim 10^{22} \mathrm{~cm}^{-2}$, while for $\operatorname{PDS} 456, L_{\text {ion }} \sim 10^{47} \mathrm{erg} \mathrm{s}^{-1}$ and $M_{\mathrm{BH}} \sim 10^{9} M_{\odot}$. Then for $\Delta t=10^{6}-10^{7} \mathrm{~s}, R \sim 4000-11,000 R_{\mathrm{g}}=0.7-1.7 \times$ $10^{18} \sim 10^{18} \mathrm{~cm}$. At this distance, and for the ionization of the gas, then the number density is $n_{\mathrm{H}} \sim 10^{7} \mathrm{~cm}^{-3}$, while the radial extent of the clouds is $\Delta R \sim 10^{15} \mathrm{~cm}$. So the outflow clouds can be fairly compact (approximately $10 R_{\mathrm{g}}$ ) and also capable of partially covering the $\mathrm{X}$-ray source.

At a distance of $R \sim 10^{18} \mathrm{~cm}$, the Keplerian velocity is $v_{\mathrm{K}} \sim 4000 \mathrm{~km} \mathrm{~s}^{-1}$. This agrees well with the measured velocity widths of the soft X-ray emission lines, with $\sigma_{\mathrm{v}}=6500 \pm 2600 \mathrm{~km} \mathrm{~s}^{-1}$ (e.g., from OBS. E), or the required velocity broadening $\left(\sigma=5000 \mathrm{~km} \mathrm{~s}^{-1}\right)$ in the XSTAR modeling. Furthermore, this matches well the velocity widths of the broad UV emission lines in PDS 456, where the FWHM of the Ly $\alpha$ and $\mathrm{C}_{\mathrm{IV}}$ lines are $12,000 \mathrm{~km} \mathrm{~s}^{-1}$ and $15,000 \mathrm{~km} \mathrm{~s}^{-1}$, respectively, and where the $\mathrm{C}$ IV profile shows one of the largest blueshifts among quasars of $\sim 5000 \mathrm{~km} \mathrm{~s}^{-1}$ (O'Brien et al. 2005; compare to Richards et al. 2002). Thus the location of the soft X-ray outflow appears coincident with the BLR in this AGN, and indeed some of the more ionized (and less dense) BLR clouds could be responsible for the soft X-ray absorption.

Alternatively, we can assume instead that the soft X-ray absorber arises from a wind with a smooth radial profile out to large distances. In that case, from integrating through the flow, then the radial extent of the wind is given by $R_{\text {smooth }}=L_{\text {ion }} / N_{\mathrm{H}} \xi \sim 10^{21} \mathrm{~cm}$ for the above outflow parameters. Such a large-scale outflow up to a kiloparsec in extent, with a rather low density (where $n_{\mathrm{H}} \sim 10 \mathrm{~cm}^{-3}$ ), would not be able to explain the variability of the absorber on timescales of weeks to months. Furthermore, the velocity widths resulting from gas on these large scales are likely to be rather small, of $\sigma \sim 100 \mathrm{~km} \mathrm{~s}^{-1}$, whereas (as seen in Figure 2) there is no narrow component of emission or absorption in PDS 456 that could be associated with a distant warm absorber. We conclude that the fast soft X-ray absorber in PDS 456 arises from clumpy gas on smaller (BLR) scales and may exist cospatially as a higher density (but lower ionization) phase of the fast accretion disk wind.

\subsection{The Soft X-Ray Emission}

The luminosity of the soft X-ray line emission can also be used to calculate the global covering factor of the outflowing gas. From the photoionization modeling, the normalization (or flux), $\kappa$, of each of the emission components is defined by XSTAR in terms of

$$
\kappa=f \frac{L_{38}}{D_{\mathrm{kpc}}^{2}}
$$

where $L_{38}$ is the ionizing luminosity in units of $10^{38} \mathrm{erg} \mathrm{s}^{-1}$, and $D_{\mathrm{kpc}}$ is the distance to the quasar in kpc. Here $f$ is the covering fraction of the gas with respect to the total solid angle, where $f=\Omega / 4 \pi$. For a spherical shell of gas, $f=1$, while $L$ is the quasar luminosity that illuminates the photoionized shell. Thus by comparing the predicted normalization $(\kappa)$ for a fully covering shell of gas illuminated by a luminosity $L$ versus the observed normalization $\left(\kappa_{\text {xstar }}\right)$ determined from the photoionization modeling, the covering fraction of the gas can be estimated. For PDS 456, with $L=10^{47} \mathrm{erg} \mathrm{s}^{-1}$ at a luminosity distance of $D=860 \mathrm{Mpc}$, for a spherical shell the expected XSTAR normalization is $\kappa=1.35 \times 10^{-3}$. Compared to the observed normalization factors reported in Table 5, the covering fraction of the strongest lower ionization (zone 1) of emitting gas is $f=0.44 \pm 0.22$.

The soft X-ray emitting (and absorbing) gas thus covers a substantial fraction of $4 \pi$ steradian, consistent with the result obtained by Nardini et al. (2015) for the high-ionization wind measured at $\mathrm{Fe} \mathrm{K}$, which was found to cover at least $2 \pi$ steradian solid angle. This is consistent with the picture of the soft X-ray outflowing gas being embedded within the wideangle high-ionization disk wind. Note that, despite the large covering factor of the soft X-ray gas, its volume-filling factor is likely smaller. This is consistent with a geometry where the absorbing clouds intercept a large enough fraction of the sightlines from the X-ray source, but individually are compact enough to occupy a small enough region in volume.

Indeed, the volume-filling factor can be estimated by considering the emission measure of the gas. To estimate the emission measure, we consider the strong O VIII RRC emission, with a flux of $\sim 7.5 \times 10^{-5}$ photons $\mathrm{cm}^{-2} \mathrm{~s}^{-1}$, as determined 
from the fit to the XMM-Newton OBS. E spectrum (see Section 5.2). This corresponds to a luminosity of $L_{\mathrm{O} \text { VIII }} \sim 6 \times 10^{51}$ photons s $^{-1}$ at the distance of PDS 456 . The recombination coefficient for $\mathrm{O}$ VIII is $\alpha_{r}=1.2 \times 10^{-11} \mathrm{~cm}^{3} \mathrm{~s}^{-1}$, for a temperature of $k T \sim 10 \mathrm{eV}$ (Verner \& Ferland 1996). The overall emission measure can then be calculated from

$$
\mathrm{EM}=\frac{L_{\mathrm{O} \text { VIII }}}{\alpha_{r} A_{\mathrm{O}} f_{i} f_{r}}
$$

where $A_{\mathrm{O}}$ is the abundance of oxygen, $f_{i}$ is the ionic fraction of the parent ion (fully ionized oxygen), and $f_{r}$ is the fraction of recombinations that occur direct to the ground state. Here we take $f_{i} \sim 0.5$ given the ionization state of the gas and $f_{r} \sim 0.3$ for the fraction of recombinations direct to ground, while an $\mathrm{O}$ abundance of $4.9 \times 10^{-4}$ is assumed (Wilms et al. 2000; Asplund et al. 2009). This gives an estimated emission measure for the soft X-ray emitting gas of EM $\sim 6 \times 10^{66} \mathrm{~cm}^{-3}$.

In comparison, we can also calculate the emission measure by assuming the gas clouds occupy a spherical region of radius $R$, with a mean density $n$ and a volume-filling factor of $V_{\mathrm{f}}$. For the emission measure:

$$
\mathrm{EM}=\int n^{2} d V \sim \frac{4}{3} \pi R^{3} V_{\mathrm{f}} n^{2}
$$

Taking a radius of $R=10^{18} \mathrm{~cm}$ (consistent with the line velocity widths) and an emitter ionization of $\log \xi \sim 3$ (for the lower-ionization zone 1) gives a density of $n=10^{8} \mathrm{~cm}^{-3}$ for the emitting clumps. By comparison of the above expression with the emission measure estimated from the line emission, this implies a volume-filling factor of $V_{\mathrm{f}} \sim 10^{-4}$, consistent with our expectations of a clumpy medium. A higher fraction of $V_{\mathrm{f}} \sim 1$ only occurs at much larger kiloparsec scales (and at correspondingly lower densities, as $n R^{2}=L_{\text {ion }} / \xi$ ), resulting in a smooth medium, but inconsistent with the observed absorption variability. Note that the clumps probably exist as quasi-spherical clouds, rather than long strands or filaments of material, in order to satisfy the criteria of a low volume-filling factor but a high covering factor.

\subsection{Outflow Energetics}

Following Nardini et al. (2015), we also estimate the mass outflow rate from

$$
\dot{M}_{\text {out }}=1.2 m_{\mathrm{p}} \times 4 \pi f v_{\text {out }} N_{\mathrm{H}} R,
$$

where $f$ is the covering fraction, and for the outflow, $v_{\text {out }}=0.2 c, N_{\mathrm{H}}=10^{22} \mathrm{~cm}^{-2}, R=10^{18} \mathrm{~cm}$ and $f \sim 0.4$ from above. As the above equation is expressed in terms of the column density, it is independent of any volume-filling factor because the observed $N_{\mathrm{H}}$ through the line of sight is the same regardless of whether the outflowing matter is smooth or clumpy. This yields a mass outflow rate of $\dot{M}_{\text {out }}=6 \times 10^{26} \mathrm{~g} \mathrm{~s}^{-1}$ (or $\sim 10 M_{\odot} \mathrm{yr}^{-1}$ ), with a corresponding kinetic power of $\dot{E}_{\mathrm{K}} \sim 10^{46} \mathrm{erg} \mathrm{s}^{-1}$ (or approximately $10 \%$ of the bolometric luminosity). This is consistent with the estimates from Nardini et al. (2015) for the highly ionized zone of the wind. This could suggest either that the lower-ionization clumps form farther out along the wind from the high- ionization matter, or that the less-ionized matter carries an energetically similar component as per the highly ionized part of the disk wind.

\subsection{The Overall View of the Wind in PDS 456}

PDS 456 is the first known AGN where it has been possible to resolve broad absorption profiles (with typical velocity widths of $\sigma \sim 5000 \mathrm{~km} \mathrm{~s}^{-1}$ ) in the soft X-ray band. In contrast, the absorption lines detected from the few fast outflows that appear to be present at soft X-rays (e.g., Gupta et al. 2013, 2015; Longinotti et al. 2015; Pounds et al. 2016) appear to result from narrower systems. Indeed, the detection of both the broad soft X-ray absorption lines and the fast P-Cygni-like profile at iron K may suggest that PDS 456 is a higherionization X-ray analog of the BAL quasars commonly known to occur in the UV (Turnshek et al. 1988; Weymann et al. 1991). Indeed, fast X-ray counterparts of the UV outflows have also been detected in some of the BAL quasars (Chartas et al. 2002, 2003). However, unlike the UV outflows, which are likely to be line driven, the X-ray outflows are generally faster and higher ionization. Indeed, they may instead be driven by other mechanisms, such as by continuum radiation, that is, Compton scattering driven winds (King \& Pounds 2003), or by magnetohydrodynamical processes (Fukumura et al. 2010).

One possibility is that the broad soft X-ray absorption lines in PDS 456 may become more apparent when the overall continuum is more absorbed. This appears to be the case in the current RGS observations, where the quasar spectrum is generally more featureless at higher flux levels, while the absorption structure becomes apparent when the continuum flux is attenuated. A similar situation may have occurred during the recent extended campaign on the nearby Seyfert 1, NGC 5548 (Kaastra et al. 2014), when broad and blueshifted UV BAL profiles unexpectedly emerged when the soft X-ray flux was heavily suppressed by a partial covering X-ray absorber. In PDS 456, the X-ray spectrum is also highly variable and similar to NGC 5548, as well as to other AGNs such as NGC 3516 (Markowitz et al. 2008; Turner et al. 2008), NGC 1365 (Risaliti et al. 2009), or ESO 323-G077 (Miniutti et al. 2014), which exhibit pronounced absorption variability at soft X-rays.

Indeed, a prolonged absorption event may have occurred in PDS 456 months prior to the 2013 XMM-Newton observations, during an extended low-flux observation with Suzaku in 2013 February-March (see Gofford et al. 2014; Matzeu et al. 2016 for details of these observations). During the 2013 Suzaku observations, the soft X-ray flux was lower by about a factor of 10 compared to the relatively unobscured level observed in XMM-Newton OBS. B just 6 months later (Figure 13). This suggests the presence of a variable, high column density obscuring medium in PDS 456, with $N_{\mathrm{H}}>10^{23} \mathrm{~cm}^{-2}$. Several months later, during the last of the current XMM-Newton observations in 2014 February (OBS. E), the soft X-ray flux below $2 \mathrm{keV}$ started to decline again when compared to OBS. B, coincident with when the broad soft X-ray absorption features emerged in the RGS spectra. The more moderate level of obscuration during OBS. E (with $N_{\mathrm{H}} \sim 10^{22} \mathrm{~cm}^{-2}$ ) likely accounts for the decrease in soft X-ray flux between OBS. B and OBS. E, while the overall level of the hard X-ray continuum above $2 \mathrm{keV}$ remained unchanged.

The overall view of the wind in PDS 456 thus appears more complex than a simple, homogeneous radial outflow. From the 


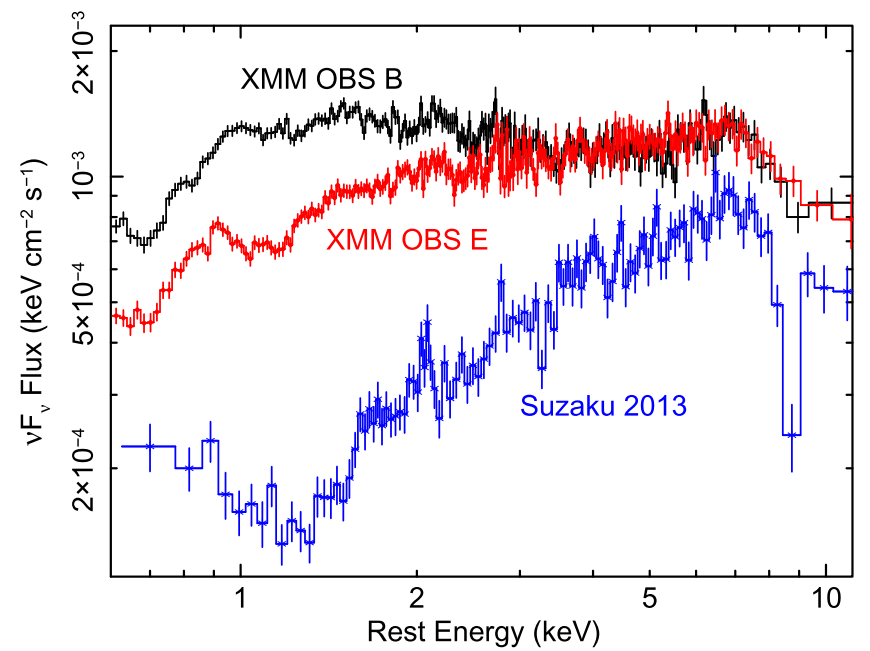

Figure 13. A comparison between the XMM-Newton EPIC MOS spectra of OBS. B (2013 September, black crosses) and OBS. E (2014 February, red circles) versus the low-flux Suzaku XIS 0+3 spectrum of PDS 456 obtained in 2013 March (blue stars). The latter spectrum corresponds to the third sequence during the 2013 February-March Suzaku campaign on PDS 456 (see Gofford et al. 2014; Matzeu et al. 2016). The $\nu F_{\nu}$ spectra have been fluxed against a $\Gamma=2$ power law. During the Suzaku 2013 observations, the quasar was at an extremely low flux, with the spectrum heavily absorbed. During the OBS. B spectrum, the quasar is relatively unobscured, and the soft X-ray flux at $1 \mathrm{keV}$ has increased by a factor of 10 over a period of 6 months. Then during OBS. E, several months later, the hard X-ray flux has remained unchanged compared to OBS. B, while the soft X-ray flux below $2 \mathrm{keV}$ declined again by a factor of $\sim 2$, consistent with the increased level of absorption revealed by the RGS spectra.

above considerations, it appears that the soft X-ray outflow represents a denser, variable, and clumpy absorber, likely embedded within the fast, less dense, higher-ionization phase of the wide-angle wind responsible for the persistent iron $\mathrm{K}$ absorption profile. The fast, high-ionization phase of the wind is likely launched from the inner accretion disk (at distances within $\sim 100 R_{\mathrm{g}}$ or $\sim 10^{16} \mathrm{~cm}$ from the black hole; see Nardini et al. 2015), while the clumps within the outflow appear to manifest over larger scales, at around $R \sim 10^{18} \mathrm{~cm}$, and are perhaps coincident with density perturbations within the wind. Indeed, hydrodynamical simulations naturally predict the existence of time-variable density variations and streamlines within the wind (Proga et al. 2000), which could then lead to the observed X-ray absorption variability. The denser clumps responsible for the soft X-ray absorption appear radially coincident with the expected location of the AGN broad emission line region, while they may also be responsible for the soft X-ray re-emission from the wind. The denser clouds may also be sufficiently compact $\left(\Delta R \sim 10 R_{\mathrm{g}}\right)$ to act as the putative partial covering X-ray absorber and may thus be responsible for the strong (order of magnitude) absorption variability seen at soft X-rays toward this quasar (Gofford et al. 2014; Matzeu et al. 2016). Indeed, in AGNs in general, a complex topology of clouds surrounding the X-ray continuum source may be able to account for the general distribution of broadband X-ray spectral shapes observed from both the type I and type II AGN populations (Tatum et al. 2013, 2016).

Future high-resolution observations may prove crucial in revealing the structure of the accretion disk wind seen in PDS 456, as well as in other quasars, and the different wind phases that are present over different scales. Furthermore, any coincidence between the UV and the soft X-ray absorber in
PDS 456 can be established by simultaneous soft X-ray observations (i.e., with XMM-Newton RGS or Chandra LETG) and the UV (via HST/COS), which, as for the case of NGC 5548, can determine how the UV line profiles respond to changes in the soft X-ray absorber.

J.N. Reeves, E. Nardini, P.T. O’Brien, and M.T. Costa acknowledge the financial support of STFC. J.N. Reeves also acknowledges NASA grant number NNX15AF12G, while T.J. Turner acknowledges NASA grant number NNX13AM27G. E. Behar received funding from the European Union's Horizon 2020 research and innovation programme under the Marie Sklodowska-Curie grant agreement no. 655324 and from the I-CORE program of the Planning and Budgeting Committee (grant number 1937/12). This research is based on observations obtained with XMM-Newton, an ESA science mission with instruments and contributions directly funded by ESA Member States and NASA.

\section{APPENDIX \\ STATISTICAL SIGNIFICANCE OF THE ABSORPTION-LINE DETECTIONS}

Here we quantify and discuss the statistical significance of the absorption-line detections in the RGS and MOS spectra, which are reported in Section 4 of the main article. As an initial guide to the significance of the line detections, Tables 2 and 3 give the $F$-test null probabilities $\left(P_{\mathrm{F}}\right)$ for the addition of each of the lines to the RGS and MOS spectra, noting that the $F$-test assumes that the prior distributions of the uncertainties are Gaussian. Nonetheless, this gives an indication of the significance level. Considering the RGS alone, the most significant lines are the broad absorption profiles in 2001 at $1.06 \mathrm{keV}$ and in the OBS. E spectrum at $1.16 \mathrm{keV}$, where $P_{\mathrm{F}}=1.3 \times 10^{-5}$ and $P_{\mathrm{F}}=9.0 \times 10^{-6}$, respectively, which exceeds the $4 \sigma$ level. The weakest two features (the emission line associated with OBS. E and the weak $850 \mathrm{eV}$ absorption line in OBS. CD) are confirmed at about the $99 \%$ level in the RGS alone.

However, when considering the MOS spectra alone, where the statistics are highest, the null probabilities are even smaller, for example, at the level of $P_{\mathrm{F}} \sim 10^{-11}$ for some of the lines, which is at the $7 \sigma$ confidence level. Indeed, the likelihood of any false positives in both RGS and MOS is very small, as they would also have to occur at a consistent energy between both detectors. For instance, the above $1.16 \mathrm{keV}$ absorption line in OBS. E has null probabilities of $P_{\mathrm{F}}=9.0 \times 10^{-6}(\mathrm{RGS})$ and $P_{\mathrm{F}}=7.4 \times 10^{-6}(\mathrm{MOS})$ for each detector, and thus the resultant probability of a false detection occurring in both detectors at the same energy is extremely small. This is also the case for the other RGS lines; indeed, when considering their multiplicative probabilities of being independently detected in both the RGS and MOS, then the line detections are all subsequently confirmed at the $5 \sigma$ or higher level.

Indeed, as a further guide to whether the spectra can be adequately accounted for by a simple baseline continuum without lines (which is the null-hypothesis case), we also quote the reduced chi-squared for the baseline model and subsequent false probability resulting from this in Tables 2 or 3 . For each spectrum, the fit statistic is extremely poor without the addition of any absorption lines to the spectra, and the baseline continuum alone is not an adequate description of the data. 
Monte Carlo simulations have then been used to assess the significance of the line detections (Protassov et al. 2002). This is essentially a means of calibrating a test statistic, whereby the prior uncertainty distribution is not known. As a test case, we ran the simulations on the OBS. E spectra, as this has the lowest continuum flux and thus may be more likely to yield a higher rate of false positives. The Monte Carlo simulations were performed independently for both the RGS and MOS exposures. For the null-hypothesis model, we adopted the bestfit baseline model for the continuum, with no absorption features, and we simulated 1000 spectra with the photon statistics expected for the same exposure times of the actual observation. In order to account for the uncertainties in the continuum model, each of the simulated spectra was then fitted with the null-hypothesis model, and a new simulation was performed with the new best fit as the continuum model; see Porquet et al. (2004) and Markowitz et al. (2008) for similar examples of applying this method.

Each of these simulated spectra was then fitted with the nullhypothesis model to obtain a baseline $\chi^{2}$ value, fitted over the full $0.4-2.0 \mathrm{keV}$ band. The spectra were systematically searched for any line-like deviations over the $0.8-1.5 \mathrm{keV}$ (rest-frame) energy range, which is the most likely band for the lines to be found in the real data. To achieve this, we added a Gaussian line component and stepped through each energy interval in the spectra, with a step size of $7 \mathrm{eV}$, and refitted the spectrum at each step. Thus, by searching the spectra for any false positives over a wide energy range, no a priori assumption is made about the initial line energy, and the Monte Carlo simulations represent a blind trial. Note that the step size over which the simulations were searched was chosen in order to match the binning used in the actual RGS and MOS spectra. We also assumed a line width of $\sim 40 \mathrm{eV}$, similar to the typical value in the actual data.

We then obtained for each RGS simulated spectrum a minimum $\chi^{2}$ and from these created a distribution of 1000 simulated values of the maximum $\left|\Delta \chi^{2}\right|$ values obtained from each spectrum (compared to the null-hypothesis model). We then constructed two independent cumulative frequency distributions of the $\left|\Delta \chi^{2}\right|$ values expected for a blind line search in both the MOS and RGS spectra.

Only one RGS fake spectrum had a $\left|\Delta \chi^{2}\right| \geqslant 17.3$, which corresponds to the lowest significance case of the reported $\left|\Delta \chi^{2}\right|$ values for the observed absorption lines in the actual OBS. E spectrum (at $1.02 \mathrm{keV}$; see Table 2). Thus the inferred rate of false detections is $P_{\mathrm{F}}=1 \times 10^{-3}$, or alternatively the statistical significance of the detection is $99.9 \%$. We then inspected the $\Delta \chi^{2}$ distribution of the MOS simulated spectra, and we found that none of the spectra has a $\left|\Delta \chi^{2}\right| \geqslant 33.4$, which is the level of the fit improvement in the actual MOS spectrum for the same $1 \mathrm{keV}$ absorption line. Indeed, the highest $\left|\Delta \chi^{2}\right|$ deviation found from the MOS simulations is 18.8; thus the false detection rate in the MOS OBS. E spectra is $P_{\mathrm{F}}<10^{-3}$. Hence, even without requiring the false detections to occur at the same energy in the MOS and RGS (i.e., within $\pm 20 \mathrm{eV}$ as above), the Monte Carlo simulations demonstrate that the absorption features in OBS.E have a combined false probability of $P_{\mathrm{F}}<10^{-6}$, given that the false detections would have to occur in both the RGS and MOS. Furthermore, given that the lines occur at the same energy in the actual RGS and MOS spectra, this confirms that the features are likely intrinsic to the AGN and are not a statistical artifact.

\section{REFERENCES}

Asplund, M., Grevesse, N., Sauval, A. J., \& Scott, P. 2009, ARA\&A, 47, 481 Behar, E. 2009, ApJ, 703, 1346

Behar, E., Kaspi, S., Reeves, J., et al. 2010, ApJ, 712, 26

Braito, V., Reeves, J. N., Gofford, J., et al. 2014, ApJ, 795, 87

Chartas, G., Brandt, W. N., \& Gallagher, S. C. 2003, ApJ, 595, 85

Chartas, G., Brandt, W. N., Gallagher, S. C., \& Garmire, G. P. 2002, ApJ, 579,169

den Herder, J. W., Brinkman, A. C., Kahn, S. M., et al. 2001, A\&A, 365, L7 Dickey, J. M., \& Lockman, F. J. 1990, ARA\&A, 28, 215

Di Matteo, T., Springel, V., \& Hernquist, L. 2005, Natur, 433, 604

Ferrarese, L., \& Merritt, D. 2000, ApJL, 539, L9

Feruglio, C., Fiore, F., Carniani, S., et al. 2015, A\&A, 583, A99

Fukumura, K., Kazanas, D., Contopoulos, I., \& Behar, E. 2010, ApJ, 715, 636

Gebhardt, K., Bender, R., Bower, G., et al. 2000, ApJL, 539, L13

Gofford, J., Reeves, J. N., Braito, V., et al. 2014, ApJ, 784, 77

Gofford, J., Reeves, J. N., Tombesi, F., et al. 2013, MNRAS, 430, 60

Grevesse, N., \& Sauval, A. J. 1998, SSRv, 85, 161

Gupta, A., Mathur, S., \& Krongold, Y. 2015, ApJ, 798, 4

Gupta, A., Mathur, S., Krongold, Y., \& Nicastro, F. 2013, ApJ, 772, 66

Hagino, K., Odaka, H., Done, C., et al. 2015, MNRAS, 446, 663

Hopkins, P. F., \& Elvis, M. 2010, MNRAS, 401, 7

Kaastra, J. S., Kriss, G. A., Cappi, M., et al. 2014, Sci, 345, 64

Kaastra, J. S., Mewe, R., Liedahl, D. A., Komossa, S., \& Brinkman, A. C. 2000, A\&A, 354, L83

Kalberla, P. M. W., Burton, W. B., Hartmann, D., et al. 2005, A\&A, 440, 775

Kallman, T., \& Bautista, M. 2001, ApJS, 133, 221

Kaspi, S., Brandt, W. N., George, I. M., et al. 2002, ApJ, 574, 643

King, A. 2003, ApJL, 596, L27

King, A. R. 2010, MNRAS, 402, 1516

King, A. R., \& Pounds, K. A. 2003, MNRAS, 345, 657

Longinotti, A. L., Krongold, Y., Guainazzi, M., et al. 2015, ApJL, 813, L39

Magorrian, J., Tremaine, S., Richstone, D., et al. 1998, AJ, 115, 2285

Markowitz, A., Reeves, J. N., \& Braito, V. 2006, ApJ, 646, 783

Markowitz, A., Reeves, J. N., Miniutti, G., et al. 2008, PASJ, 60, 227

Matzeu, G. A., Reeves, J. N., Nardini, E., et al. 2016, MNRAS, 458, 1311

Miniutti, G., Sanfrutos, M., Beuchert, T., et al. 2014, MNRAS, 437, 1776

Nardini, E., Reeves, J. N., Gofford, J., et al. 2015, Sci, 347, 860

O'Brien, P. T., Reeves, J. N., Simpson, C., \& Ward, M. J. 2005, MNRAS, 360, L25

Porquet, D., Reeves, J. N., Uttley, P., \& Turner, T. J. 2004, A\&A, 427, 101

Pounds, K., Lobban, A., Reeves, J. N., \& Vaughan, S. 2016, MNRAS, 457, 2951

Pounds, K. A. 2014, MNRAS, 437, 3221

Pounds, K. A., Reeves, J. N., King, A. R., et al. 2003, MNRAS, 345, 705

Proga, D., Stone, J. M., \& Kallman, T. R. 2000, ApJ, 543, 686

Protassov, R., van Dyk, D. A., Connors, A., Kashyap, V. L., \& Siemiginowska, A. 2002, ApJ, 571, 545

Reeves, J. N., Braito, V., Gofford, J., et al. 2014, ApJ, 780, 45

Reeves, J. N., O'Brien, P. T., Braito, V., et al. 2009, ApJ, 701, 493

Reeves, J. N., O'Brien, P. T., Vaughan, S., et al. 2000, MNRAS, 312, L17

Reeves, J. N., O'Brien, P. T., \& Ward, M. J. 2003, ApJL, 593, L65

Richards, G. T., Vanden Berk, D. E., Reichard, T. A., et al. 2002, AJ, 124, 1

Risaliti, G., Salvati, M., Elvis, M., et al. 2009, MNRAS, 393, L1

Sako, M., Kahn, S. M., Behar, E., et al. 2001, A\&A, 365, L168

Silk, J., \& Rees, M. J. 1998, A\&A, 331, L1

Simpson, C., Ward, M., O’Brien, P., \& Reeves, J. 1999, MNRAS, 303, L23

Tatum, M. M., Turner, T. J., Miller, L., et al. 2016, ApJ, 818, 12

Tatum, M. M., Turner, T. J., Miller, L., \& Reeves, J. N. 2013, ApJ, 762, 80

Tombesi, F., Cappi, M., Reeves, J. N., et al. 2010, A\&A, 521, A57

Tombesi, F., Cappi, M., Reeves, J. N., et al. 2013, MNRAS, 430, 1102

Tombesi, F., Meléndez, M., Veilleux, S., et al. 2015, Natur, 519, 436

Torres, C. A. O., Quast, G. R., Coziol, R., et al. 1997, ApJL, 488, L19

Turner, T. J., Reeves, J. N., Kraemer, S. B., \& Miller, L. 2008, A\&A, 483, 161

Turnshek, D. A., Grillmair, C. J., Foltz, C. B., \& Weymann, R. J. 1988, ApJ, 325,651

Verner, D. A., \& Ferland, G. J. 1996, ApJS, 103, 467

Weymann, R. J., Morris, S. L., Foltz, C. B., \& Hewett, P. C. 1991, ApJ, 373,23

Wilms, J., Allen, A., \& McCray, R. 2000, ApJ, 542, 914

Yun, M. S., Reddy, N. A., Scoville, N. Z., et al. 2004, ApJ, 601, 723 